\begin{tabular}{|c|c|}
\hline Title & $\begin{array}{l}\text { Reduction in higher order effects on logic functions of asymmetric nonlinear optical fiber couplers by bandwidth } \\
\text { limited amplification }\end{array}$ \\
\hline Author(s) & Koshiba, M.; Kojima, T.; Tsuji, Y .; Eguchi, M. \\
\hline Citation & $\begin{array}{l}\text { Journal of Lightwave Technology, 15(2), 279-287 } \\
\text { https://doi.org/10.1109/50.554378 }\end{array}$ \\
\hline Issue Date & 1997-02 \\
\hline Doc URL & http:/hdl.handle.net/2115/6044 \\
\hline Rights & $\begin{array}{l}\text { @1997 IEEE. Personal use of this material is permitted. However, permission to reprint/republish this material for } \\
\text { advertising or promotional purposes or for creating new collective works for resale or redistribution to servers or lists, } \\
\text { or to reuse any copyrighted component of this work in other works must be obtained from the IEEE. } \\
\text { IEEE, Journal of Lightwave Technology, 15(2), 1997, p279-287 }\end{array}$ \\
\hline Type & article \\
\hline File Information & JLT15_2.pdf \\
\hline
\end{tabular}

Instructions for use 


\title{
Reduction in Higher Order Effects on Logic Functions of Asymmetric Nonlinear Optical Fiber Couplers by Bandwidth Limited Amplification
}

\author{
Masanori Koshiba, Senior Member, IEEE, Takashi Kojima, Yasuhide Tsuji, \\ and Masashi Eguchi, Member, IEEE
}

\begin{abstract}
Higher order effects such as third-order dispersion, shock effect, and soliton self-frequency shift on all-optical logic gates that use asymmetric nonlinear optical fiber couplers are investigated with the beam propagation method, and it is found that third-order dispersion seriously affects the operations of logic devices. In contrast to the case of nonvanishing thirdorder dispersion, the radiation is stimulated, and consequently the operations of the logic devices are degraded. In order to diminish the degradation, the bandwidth limited amplification is introduced. The logic functions can be improved by trapping only the soliton components in the finite gain-bandwidth.
\end{abstract}

Index Terms-All-optical logic gate, nonlinear directional coupler, optical soliton.

\section{INTRODUCTION}

S OLITON pulses in optical fibers [1]-[3] whose transmission loss is effectively compensated by an erbium-doped fiber amplifier (EDFA) [4] are currently a subject of great interest, because of their potential applications in optical signal processing and transmission [5]. Optical solitons are also being considered for potential use in all-optical switching and logic. The phenomenon of nonlinear directional coupling has been widely investigated for applications to all-optical ultrafast switching [6]-[15], and recently, all-optical ultrafast logic functions that use an asymmetric nonlinear directional coupler (NLDC) have been numerically demonstrated [16]. Moreover, the switching response of asymmetric nonlinear couplers has been experimentally investigated [17]-[19]. In such an asymmetric coupler, the output power tends to appear in the waveguide with the stronger Kerr effect when only one waveguide is excited. When both waveguides are excited, the power distribution at the output end depends on the relative phase of the input signals. The asymmetric nonlinear directional couplers make it possible to implement various logic gates such as AND, OR, and XOR gates [16], but the coupler length becomes long when picosecond solitons are used. To reduce this coupler length, shorter solitons must

Manuscript received August 2, 1995; revised July 29, 1996.

M. Koshiba, T. Kojima, and Y. Tsuji are with the Division of Electronics and Information Engineering, Hokkaido University, Sapporo 060, Japan.

M. Eguchi is with the Department of Electrical and Electronics Engineering, Muroran Institute of Technology, Muroran 050, Japan.

Publisher Item Identifier S 0733-8724(97)01380-7. be used. However, when subpicosecond-femtosecond solitons are used, higher order effects such as third-order dispersion (TOD), shock effect, and soliton self-frequency shift (SSFS) may degrade the operations of the logic devices. Although the effects of TOD on symmetric NLDC's have been investigated recently [11], the higher order effects on asymmetric NLDC's have not been discussed yet.

In this paper the higher order effects on all-optical logic gates that use asymmetric nonlinear optical fiber couplers are investigsted with the beam propagation method (BPM), and it is found that TOD strongly affects the operations of logic devices. In contrast to the case of nonvanishing TOD, the radiation is stimulated, and consequently the operations of logic devices are degraded. In order to diminish the degradation, the bandwidth limited amplification [9], [20], [21] is introduced. It has been shown that the higher order effects can be suppressed by the bandwidth limited amplification. The logic functions can be improved by trapping only the soliton components in the finite gain-bandwidth. The effects of fiber loss and walk-off are also discussed.

\section{THEORETICAL MODEL}

Coupled nonlinear Schrödinger equations (NLSE's) with higher order effects that describe subpicosecond-femtosecond soliton-propagation in two channels are given by

$$
\begin{aligned}
& -j \frac{\partial u}{\partial \xi}+\frac{1}{2} \frac{\partial^{2} u}{\partial \tau^{2}}+|u|^{2} u+\kappa v+j G\left(u+\mu \frac{\partial^{2} u}{\partial \tau^{2}}\right) \\
& -j \Gamma u+j \nu_{1} \frac{\partial^{3} u}{\partial \tau^{3}}-j \nu_{2} \frac{\partial\left(|u|^{2} u\right)}{\partial \tau}-\nu_{3} \frac{\partial|u|^{2}}{\partial \tau} u=0 \\
& -j \frac{\partial v}{\partial \xi}+\frac{1}{2} \frac{\partial^{2} v}{\partial \tau^{2}}+q|v|^{2} v+\kappa u+j G\left(v+\mu \frac{\partial^{2} v}{\partial \tau^{2}}\right) \\
& -j \Gamma v+j \nu_{1} \frac{\partial^{3} v}{\partial \tau^{3}}-j \nu_{2} \frac{\partial\left(|v|^{2} v\right)}{\partial \tau}-\nu_{3} \frac{\partial|v|^{2}}{\partial \tau} v=-j \delta \frac{\partial v}{\partial \tau}
\end{aligned}
$$

where $u$ and $v$ are the normalized pulse amplitudes of the two channels, $\kappa$ is the coupling coefficient, and the normalized coordinates, $\xi, \tau$, the normalized gain and bandwidth of EDFA, $G, \mu$, and the coefficients $\Gamma, \nu_{1}, \nu_{2}, \nu_{3}$, and $\delta$ which are, respectively, related to the fiber loss, TOD, shock effect, SSFS, 


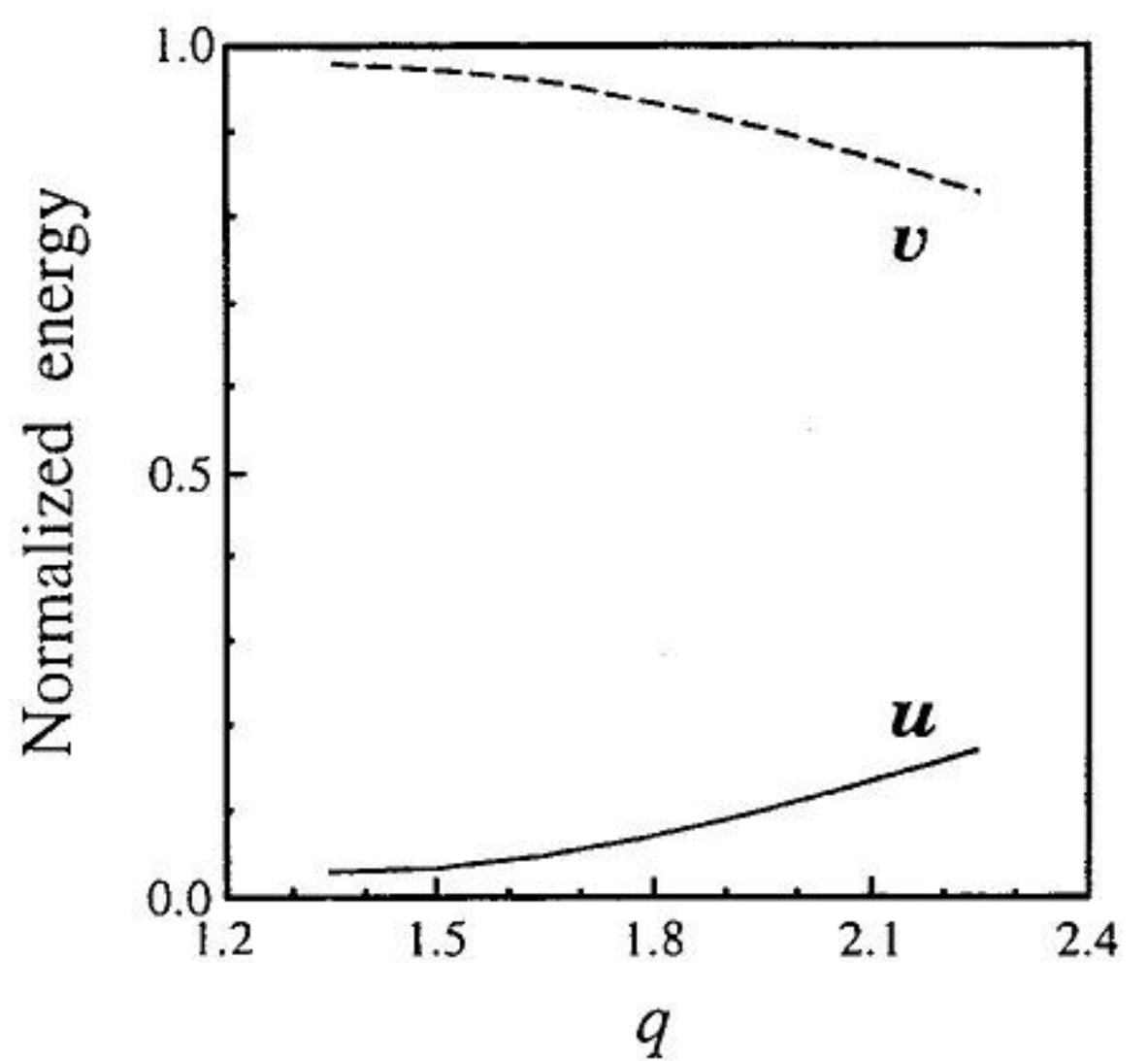

(a)

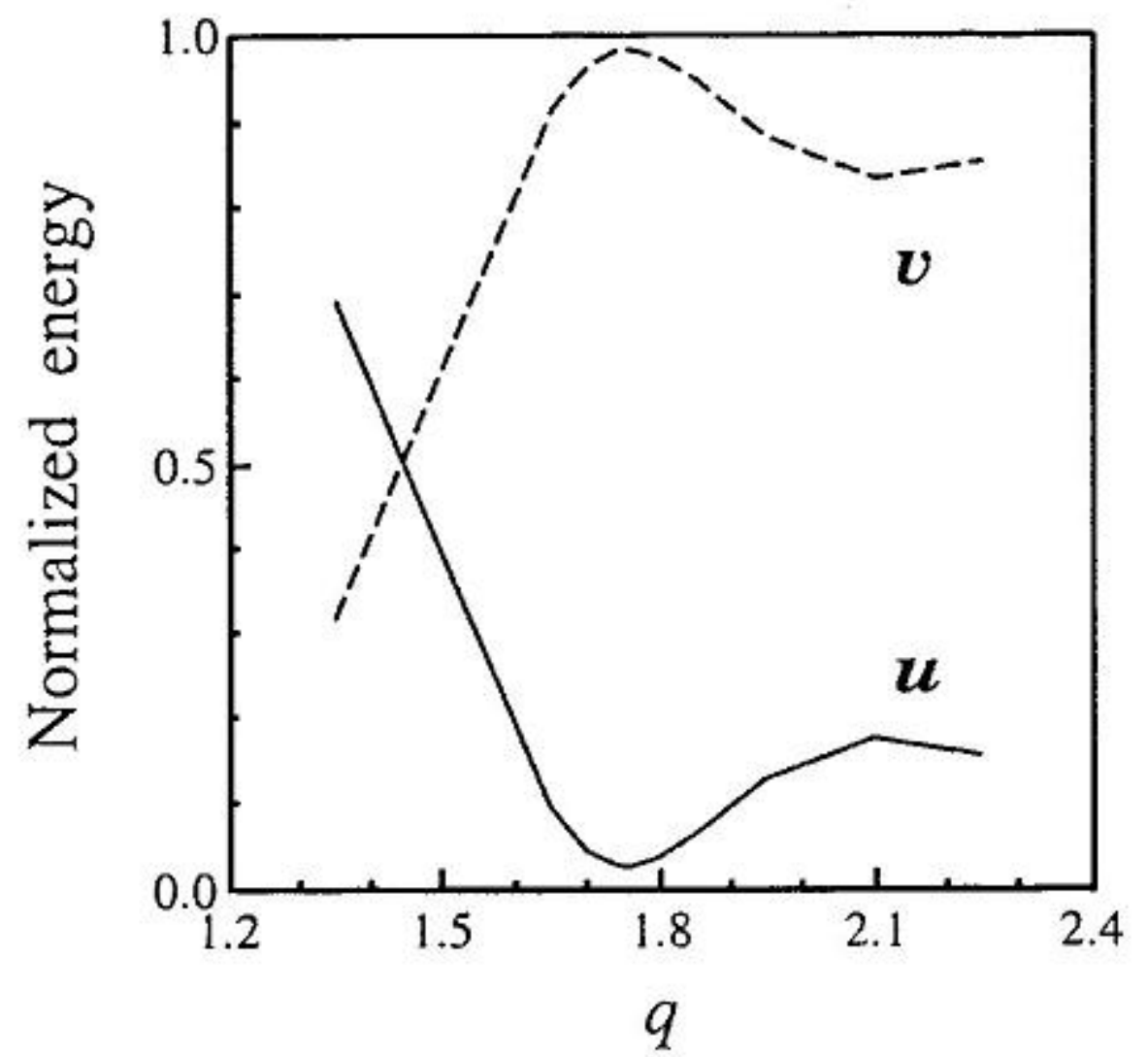

(b)

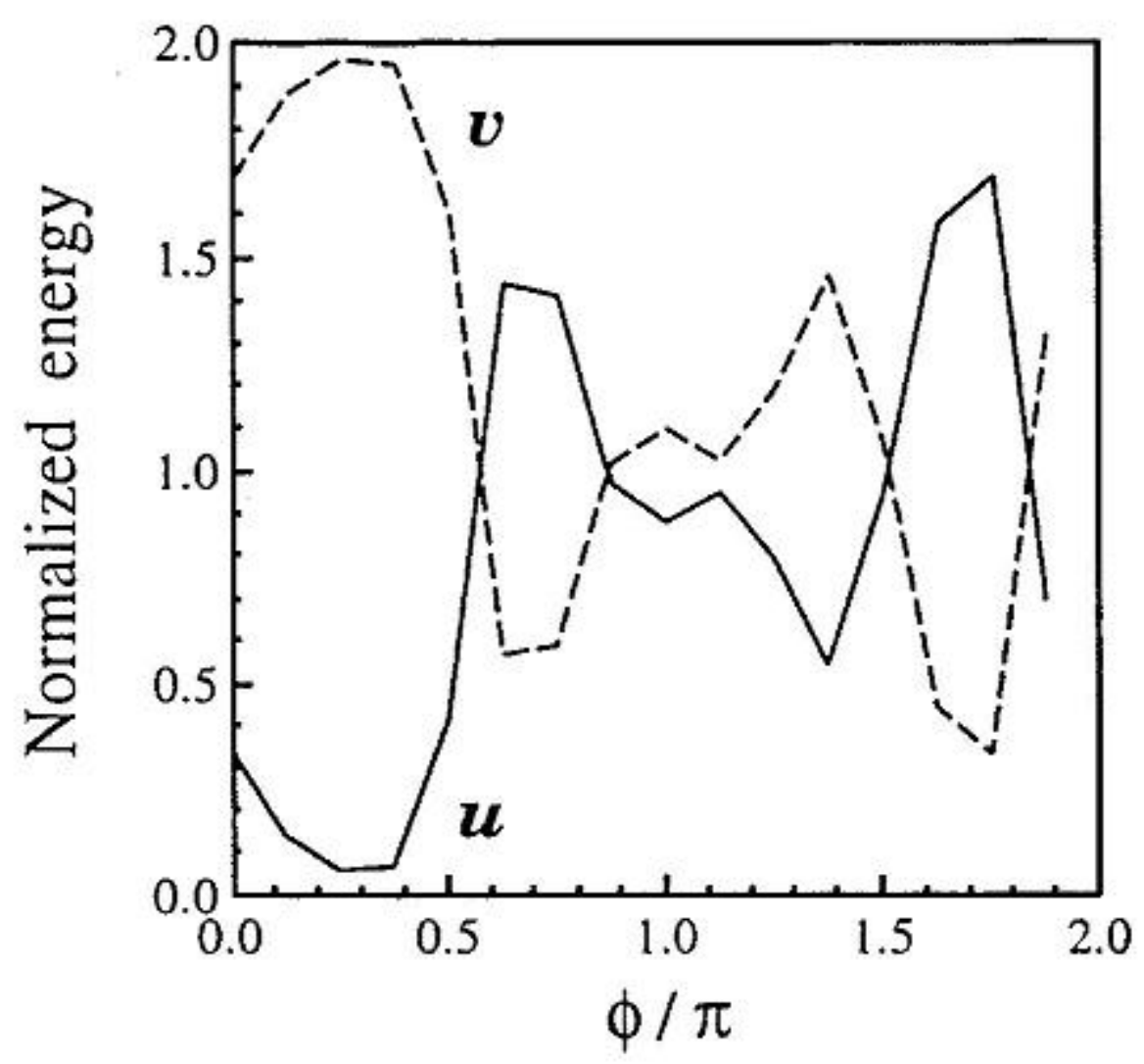

(c)

Fig. 1. Switching characteristics in the unperturbed case: (a) $A_{u}=1, A_{v}=0$, and $\phi=0$, (b) $A_{u}=0, A_{v}=1$, and $\phi=0$, and (c) $A_{u}=A_{v}=1$, and $q=1.75$.

and walk-off effect are defined as follows:

$$
\begin{aligned}
\xi & =\frac{\pi}{2} \frac{z}{Z_{s}} \\
\tau & =\frac{1}{T_{\mathrm{FWHM}}}\left(t-\frac{z}{v_{g}}\right) \times 1.76274 \\
G & =\frac{g T_{\mathrm{FWHM}}^{2}}{|\sigma| \lambda^{2}} \times 6.9793 \times 10^{-2} \\
\mu & =\left(\frac{\lambda^{2}}{\lambda_{g} T_{\mathrm{FWHM}}}\right)^{2} \times 1.75148 \\
\Gamma & =\frac{2}{\pi} Z_{s} \frac{\alpha}{20000 \log _{10} e} \\
\nu_{1} & =-\frac{\sigma_{\lambda} \lambda^{2}}{|\sigma| T_{\mathrm{FWHM}}} \times 1.5597 \times 10^{-1} \\
\nu_{2} & =\frac{\lambda}{T_{\mathrm{FWHM}}} \times 1.87162 \times 10^{-3} \\
\nu_{3} & =\frac{T_{r}}{T_{\mathrm{FWHM}}} \times 1.76274 \times 10^{-3}
\end{aligned}
$$

(3) with

(5) Here $z(\mathrm{~km})$ is the spatial coordinate, $t$ (ps) is the temporal coordinate, $v_{g}(\mathrm{ps} / \mathrm{km})$ is the group velocity of $u$ pulse $T_{\text {FWHM }}$ (ps) is the full width at half maximum (FWHM) of the pulse intensity, $\lambda(\mu \mathrm{m})$ is the carrier wavelength, 0 $(\mathrm{ps} /(\mathrm{km} \cdot \mathrm{nm}))$ is the group velocity dispersion (GVD) and positive in the anomalous-dispersion regime, $\sigma_{\lambda}\left(\mathrm{ps} /\left(\mathrm{km} \cdot \mathrm{nm}^{2}\right)\right)$ is the third-order dispersion, $g(\mathrm{~dB} / \mathrm{km})$ is the gain per unit length, $\lambda_{g}(\mathrm{~nm})$ is the gain bandwidth (FWHM), $\alpha(\mathrm{dB} / \mathrm{km})$ is the fiber loss, $T_{r}$ (fs) is the finite response time of the nonlinear refractive-index or the Raman process, $T_{d}$ is the normalized delay time difference between $u$ - and $v$-channels, $Z_{s}(\mathrm{kmi})$ corresponding to $\xi=\pi / 2$ is called the soliton period, and the coupling and the asymmetric natures of the two channels are, 


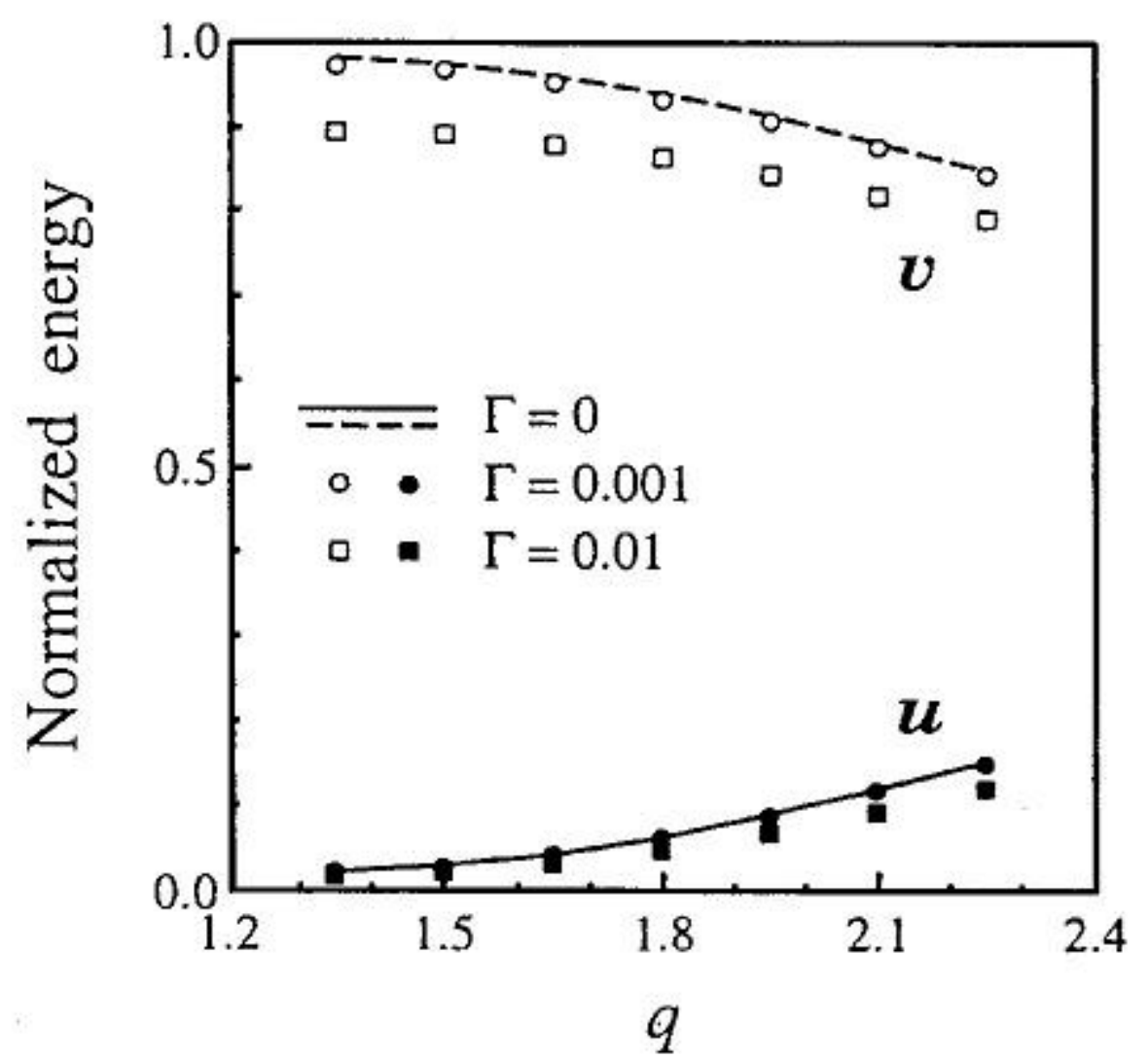

(a)

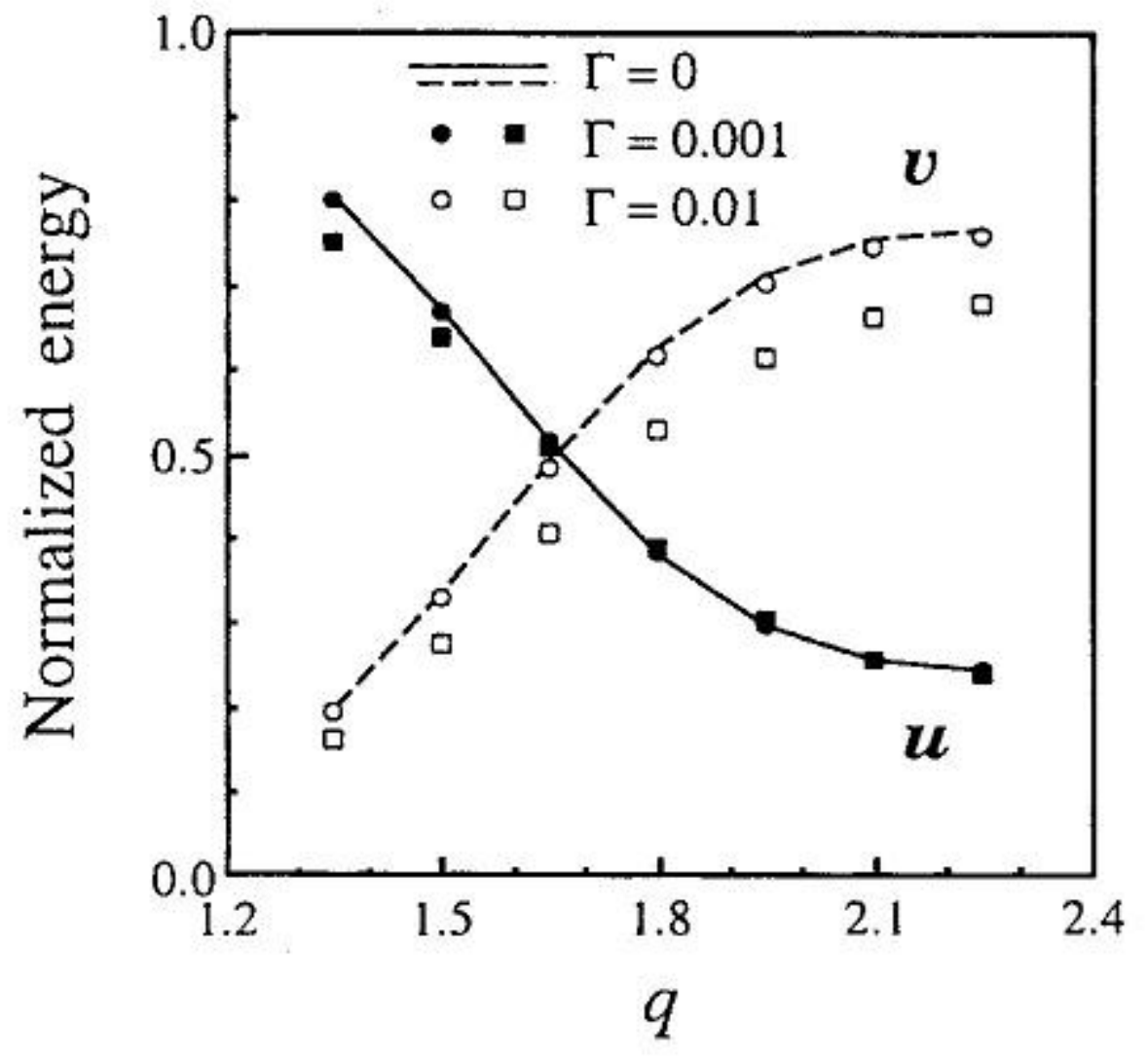

(b)

Fig. 2. Switching characteristics in the perturbed case: (a) $A_{u}=1, A_{v}=0$, and $\phi=0$ and (b) $A_{u}=0, A_{v}=1$, and $\phi=0$.

respectively, expressed by the coefficients, $\kappa$ and $q$. In (1) and (2) no GVD difference between $u$ - and $v$-channels is assumed, and the asymmetry of the higher order effects is neglected.

\section{ANALYSIS METHOD}

Coupled NLSE's are solved by using the BPM with initial pulse forms given by

$$
\begin{aligned}
& u(\xi=0, \tau)=A_{u} \operatorname{sech} \tau \\
& v(\xi=0, \tau)=A_{v} \exp (j \phi) \operatorname{sech} \tau
\end{aligned}
$$

where $A_{u}$ and $A_{v}$ are the amplitudes of $u$ and $v$ pulses, respectively, and $\phi$ is the relative phase of the two pulses.

To apply the BPM to coupled NLSE's, we rewrite (1) and (2) as

$$
\frac{\partial u}{\partial \xi}=\left(D_{u}+N_{u}\right) u-j \kappa v
$$

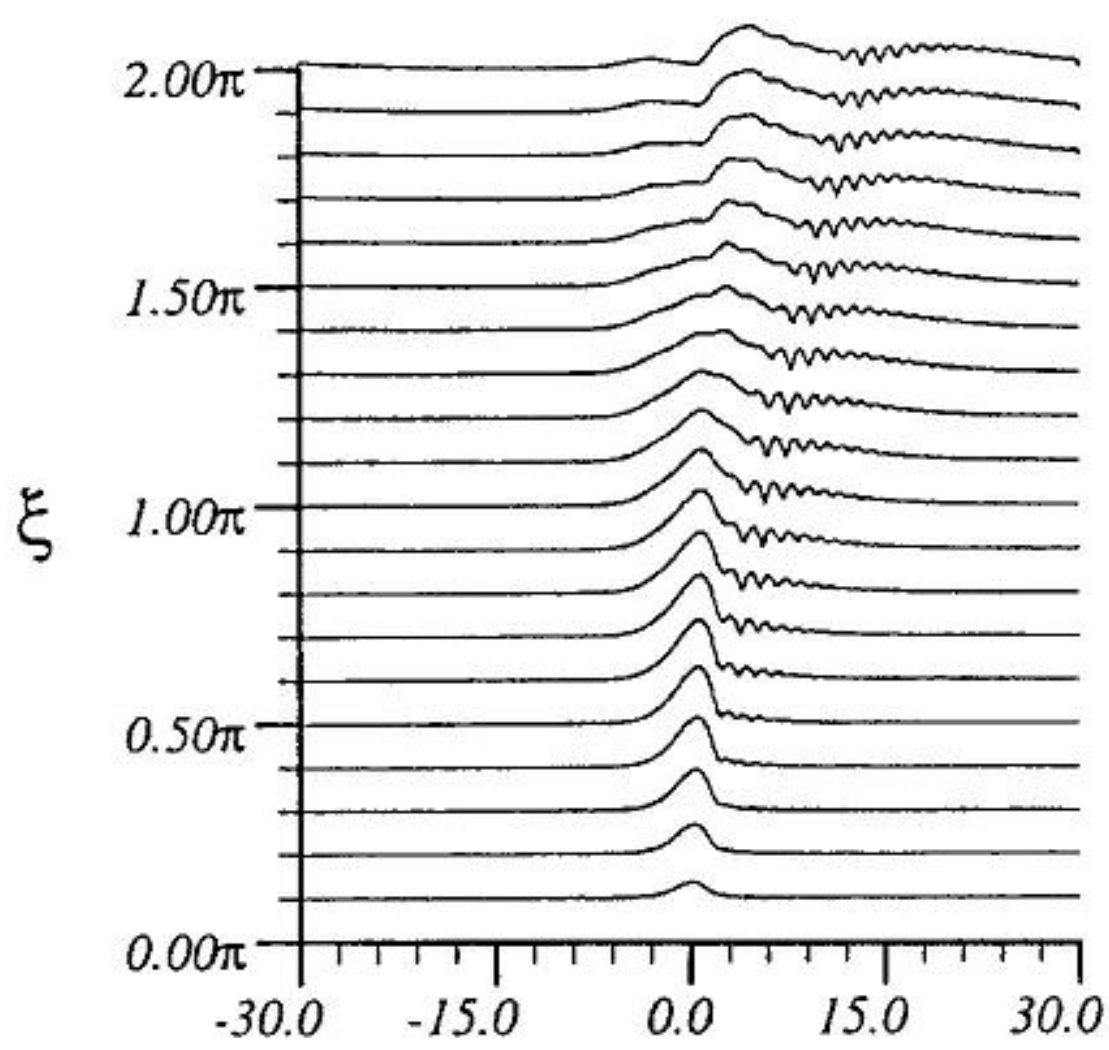

\section{$\tau$}

(a)

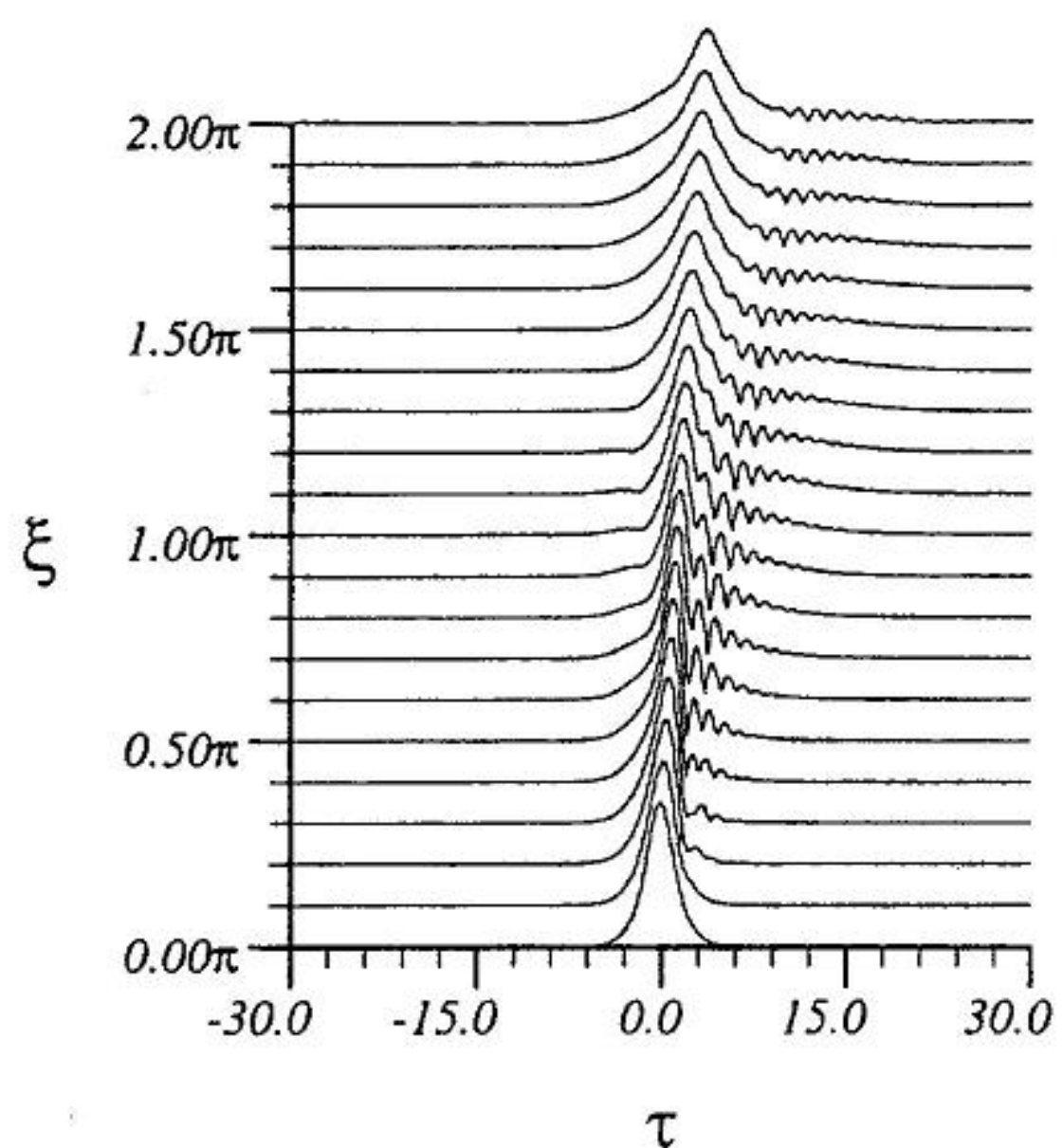

(b)

Fig. 3. Waveform changes in the perturbed case $\left(A_{u}=0, A_{v}=1\right.$, and $\phi=0$ ): (a) $u$-channel and (b) $v$-channel.

$$
\frac{\partial v}{\partial \xi}=\left(D_{v}+N_{v}\right) v-j \kappa u
$$

with

$$
\begin{aligned}
D_{u}= & -j \frac{1}{2} \frac{\partial^{2}}{\partial \tau^{2}}+G \mu \frac{\partial^{2}}{\partial \tau^{2}}+\nu_{1} \frac{\partial^{3}}{\partial \tau^{3}} \\
D_{v}= & -j \frac{1}{2} \frac{\partial^{2}}{\partial \tau^{2}}+G \mu \frac{\partial^{2}}{\partial \tau^{2}}+\nu_{1} \frac{\partial^{3}}{\partial \tau^{3}}+\delta \frac{\partial}{\partial \tau} \\
N_{u}= & -j|u|^{2}+G-\Gamma-\nu_{2}\left[\left(|u|^{2}\right)_{\tau}+u^{*}(u)_{\tau}\right] \\
& +j \nu_{3}\left(|u|^{2}\right)_{\tau} \\
N_{v}= & -j q|v|^{2}+G-\Gamma-\nu_{2}\left[\left(|v|^{2}\right)_{\tau}+v^{*}(v)_{\tau}\right] \\
& +j \nu_{3}\left(|v|^{2}\right)_{\tau}
\end{aligned}
$$

where $(\cdot)_{\tau}$ means the numerical differentation with respect to $\tau$ 


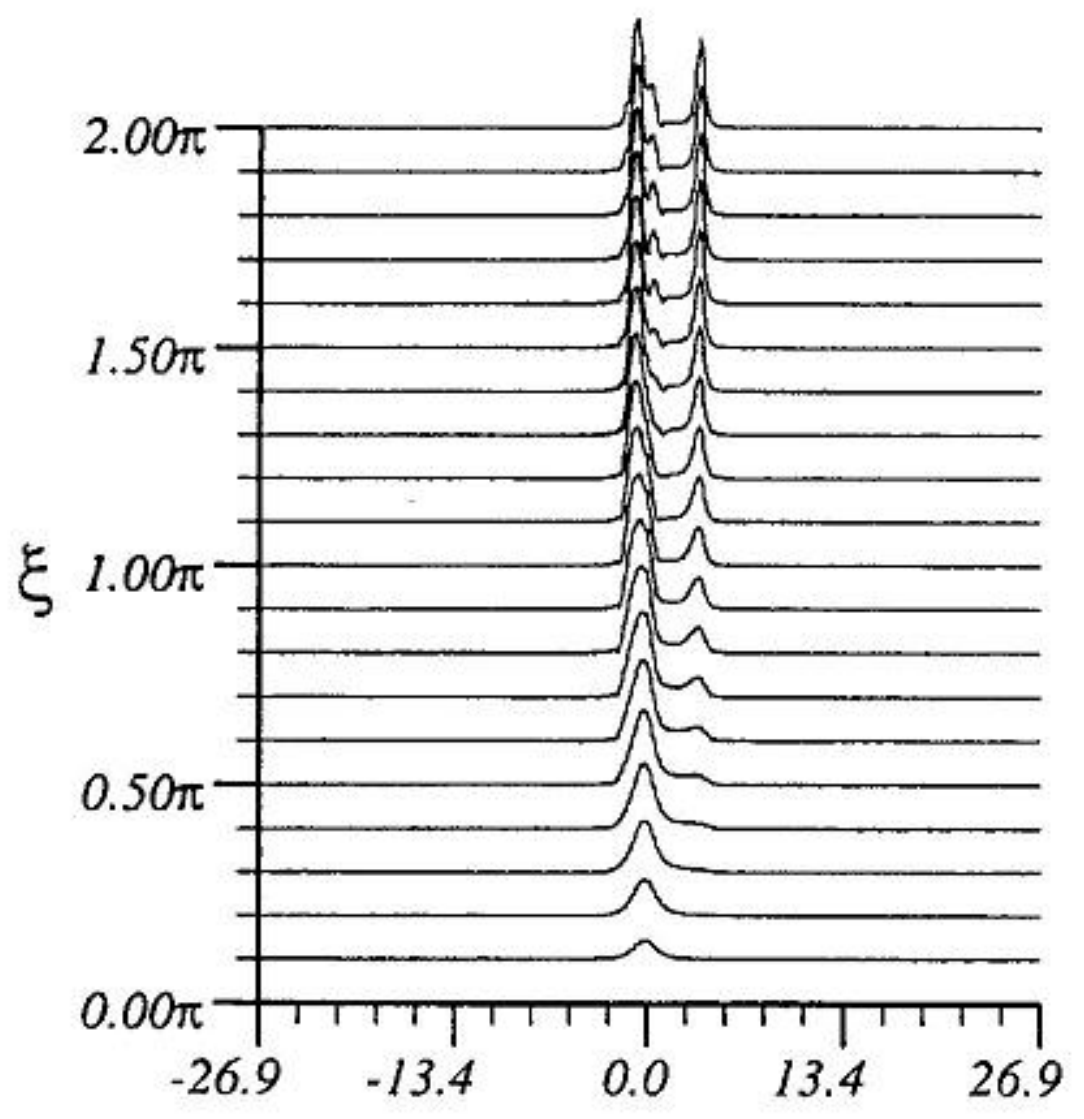

$\left[\omega-\omega_{0}\right] T$

(a)

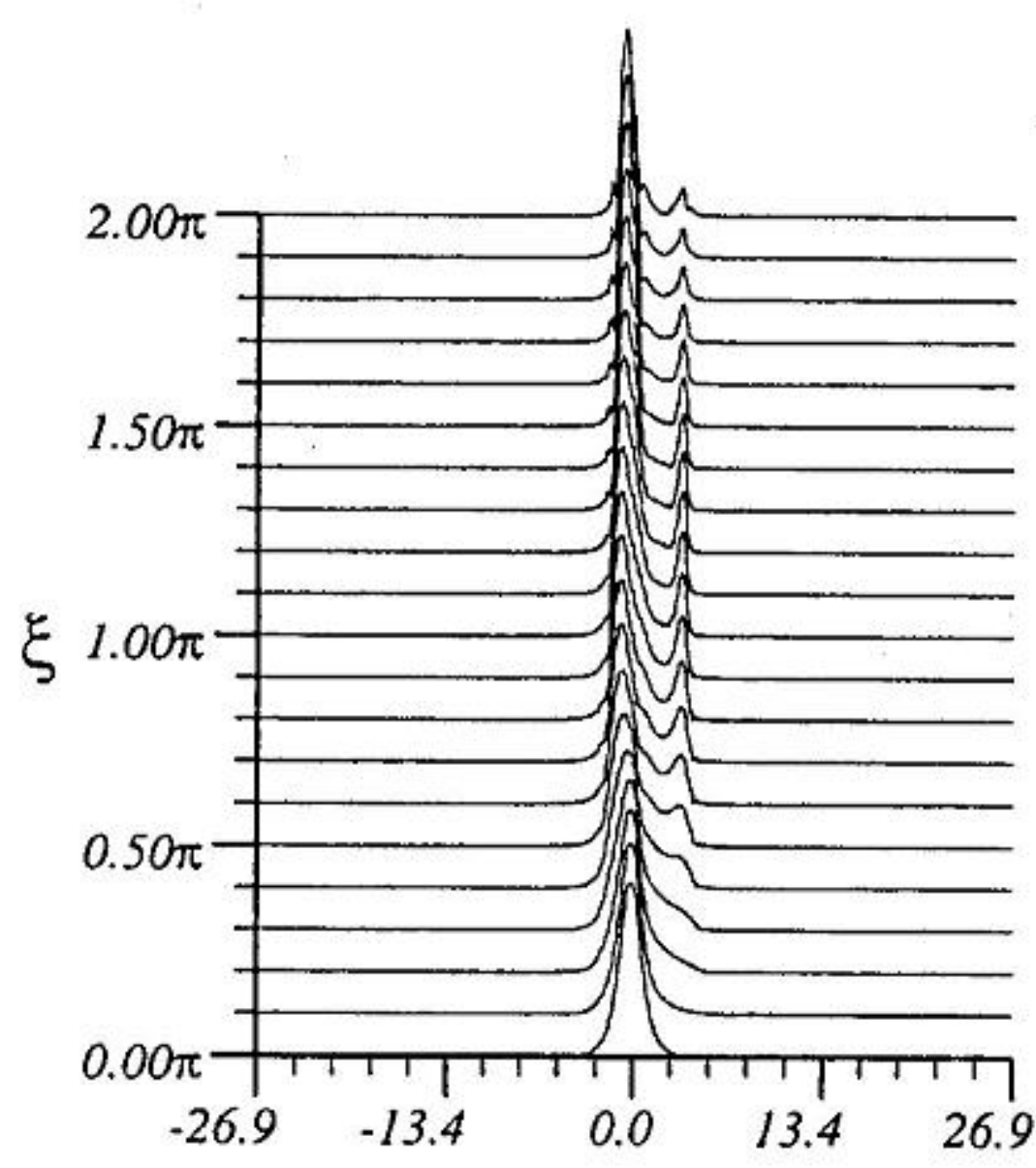

$\left[\omega-\omega_{0}\right] T$

(b)

Fig. 4. Spectrum changes in the perturbed case $\left(A_{u}=0, A_{v}=1\right.$, and $\phi=0$ ): (a) $u$-channel and (b) $v$-channel.

From (15) and (16) the following successive equations are derived:

$$
\begin{aligned}
u(\xi+\Delta \xi, \tau)= & \exp \left(D_{u} \Delta \xi / 2\right) \exp \left(N_{u} \Delta \xi\right) \exp \left(D_{u} \Delta \xi / 2\right) \\
& \times u(\xi, \tau)-j \kappa v(\xi+\Delta \xi / 2, \tau) \\
v(\xi+\Delta \xi, \tau)= & \exp \left(D_{v} \Delta \xi / 2\right) \exp \left(N_{v} \Delta \xi\right) \exp \left(D_{v} \Delta \xi / 2\right) \\
& \times v(\xi, \tau)-j \kappa u(\xi+\Delta \xi / 2, \tau) .
\end{aligned}
$$

In the BPM, the pulse forms at $\xi=\xi+\Delta \xi$ can be predicted from those at $\xi=\xi$.
TABLE I

LOGIC FUNCTIONS

\begin{tabular}{ccccc}
\hline \multirow{2}{*}{ Parameters } & \multicolumn{3}{c}{ Input } & \multicolumn{2}{c}{ Output } \\
\cline { 2 - 5 } & $E_{u}$ & $E_{v}$ & $E_{u}$ & $E_{v}$ \\
\hline$\nu_{1}=\nu_{2}=\nu_{3}=0$ & 0 & 0 & 0 & 0 \\
$G=\mu=\Gamma=0$ & 1 & 0 & $0(0.06)$ & $1(0.94)$ \\
$q=1.75$ & 0 & 1 & $0(0.02)$ & $1(0.98)$ \\
$\phi=0.865 \pi$ & 1 & 1 & $1(1.00)$ & $1(0.99)$ \\
\hline$\nu_{1}=0.2, \nu_{2}=0.01, \nu_{3}=0.04$ & 0 & 0 & 0 & 0 \\
$G=0.07, \mu=1.5, \Gamma=0$ & 1 & 0 & $0(0.22)$ & $1(1.19)$ \\
$q=1.95$ & 0 & 1 & $0(0.19)$ & $1(0.96)$ \\
$\phi=0.800 \pi$ & 1 & 1 & $1(1.12)$ & $1(1.12)$ \\
\hline$\nu_{1}=0.2, \nu_{2}=0.01, \nu_{3}=0.04$ & 0 & 0 & 0 & 0 \\
$G=0.07, \mu=1.5, \Gamma=0.01$ & 1 & 0 & $0(0.17)$ & $1(1.14)$ \\
$q=1.95$ & 0 & 1 & $0(0.21)$ & $1(0.85)$ \\
$\phi=0.820 \pi$ & 1 & 1 & $1(1.06)$ & $1(1.04)$ \\
\hline
\end{tabular}

\section{LOGIC FUNCTIONS}

In this section, we show the logic functions that use asym metric nonlinear fiber couplers, and assume $\kappa=0.331$ [16] which yields the normalized coupling length, $\xi_{c}=\pi / 2 \kappa \simeq$ $1.5 \pi$. To investigate the operation principle of asymmetric nonlinear fiber couplers, in Sections IV-A to -D we assume no walk-off effect, $\delta=0$.

\section{A. Unperturbed Case}

In order to confirm the validity of our analysis method, we consider the unperturbed case; $\nu_{1}=\nu_{2}=\nu_{3}=G=\mu$ e $\Gamma=0$.

Fig. 1 shows the normalized energy in $u$-channel denoted by the solid line and in $v$-channel denoted by the dashed line at the coupling length. In Fig. 1(a) or (b), the fundamental soliton is inputted to $u$ - or $v$-channel, respectively, and we assume $\phi=0$.

In the former case, the optical soliton input to $u$-channel coupled to $v$-channel, irrespective of the values of $q$. In th latter case, on the other hand, as the values of $q$ become larges the soliton inputted to $v$-channel remains in $v$-channel without being coupled. The contrast of output levels between $u$ - and $v$-channels becomes maximum when $q \simeq 1$.75. In Fig. 11 (c) the fundamental solitons are inputted to both channels, and we assume $q=1.75$. The output levels in $u$ - and $v$-channels are sensitive to the values of $\phi$ and are almost the same whel: $\phi=0.570 \pi, 0.865 \pi, 1.510 \pi$, and $1.808 \pi$.

From these results it is found that, when $q=1.75$ $\phi=0.865 \pi, u$ - and $v$-channels act like AND and OR gat respectively, as shown in Table I, where the numbers parentheses reflect the real simulation results. These resu agree well with those of Yang [16].

\section{B. Perturbed Case}

Here, we investigate the higher order effects on the ople ations of lossless $(\alpha=0)$ logic devices without EDFAa assume $T_{\mathrm{FWHM}}=0.25 \mathrm{ps}, \lambda=1.55 \mu \mathrm{m}$, and $T_{r}=5.9$ 


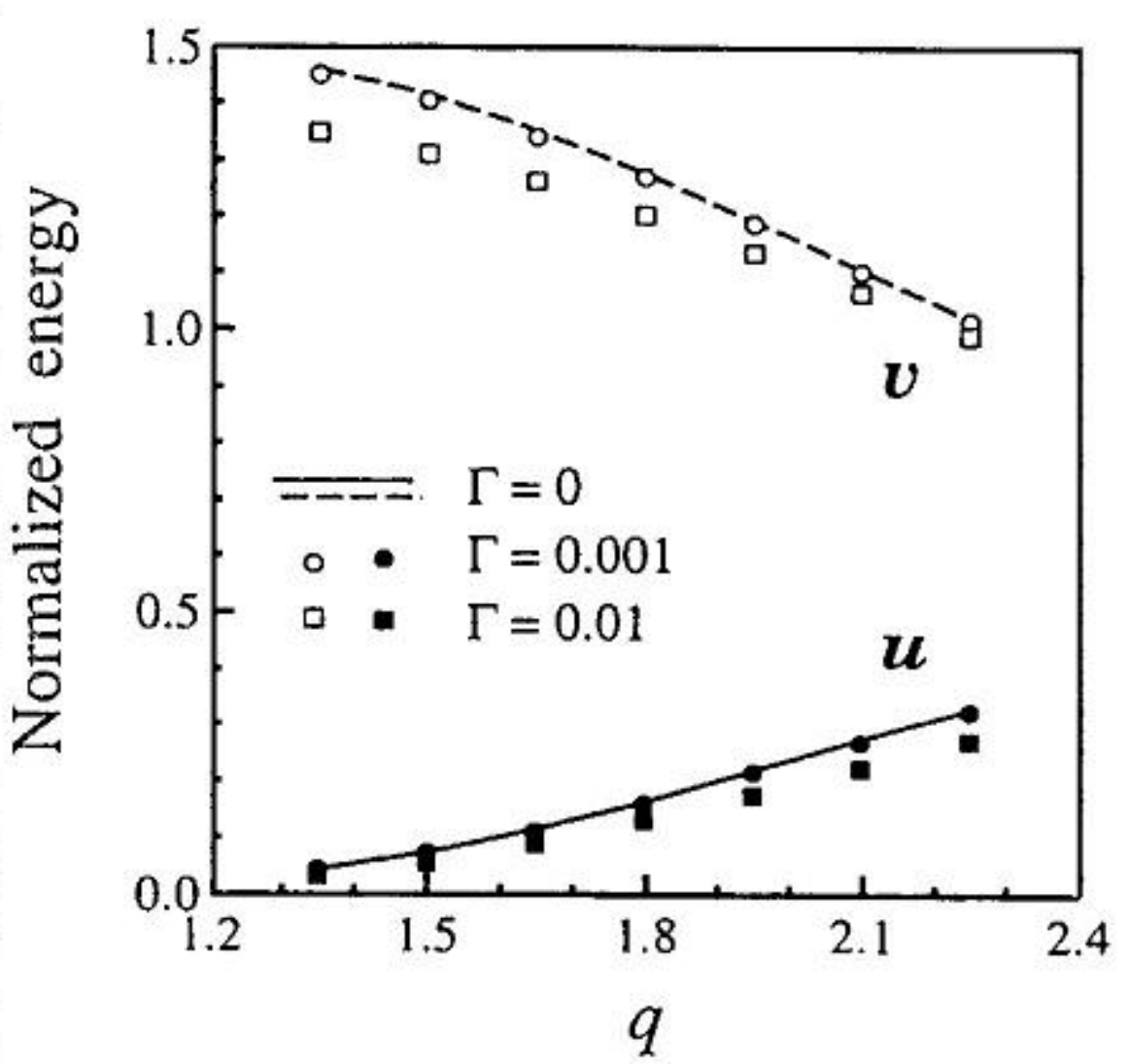

(a)

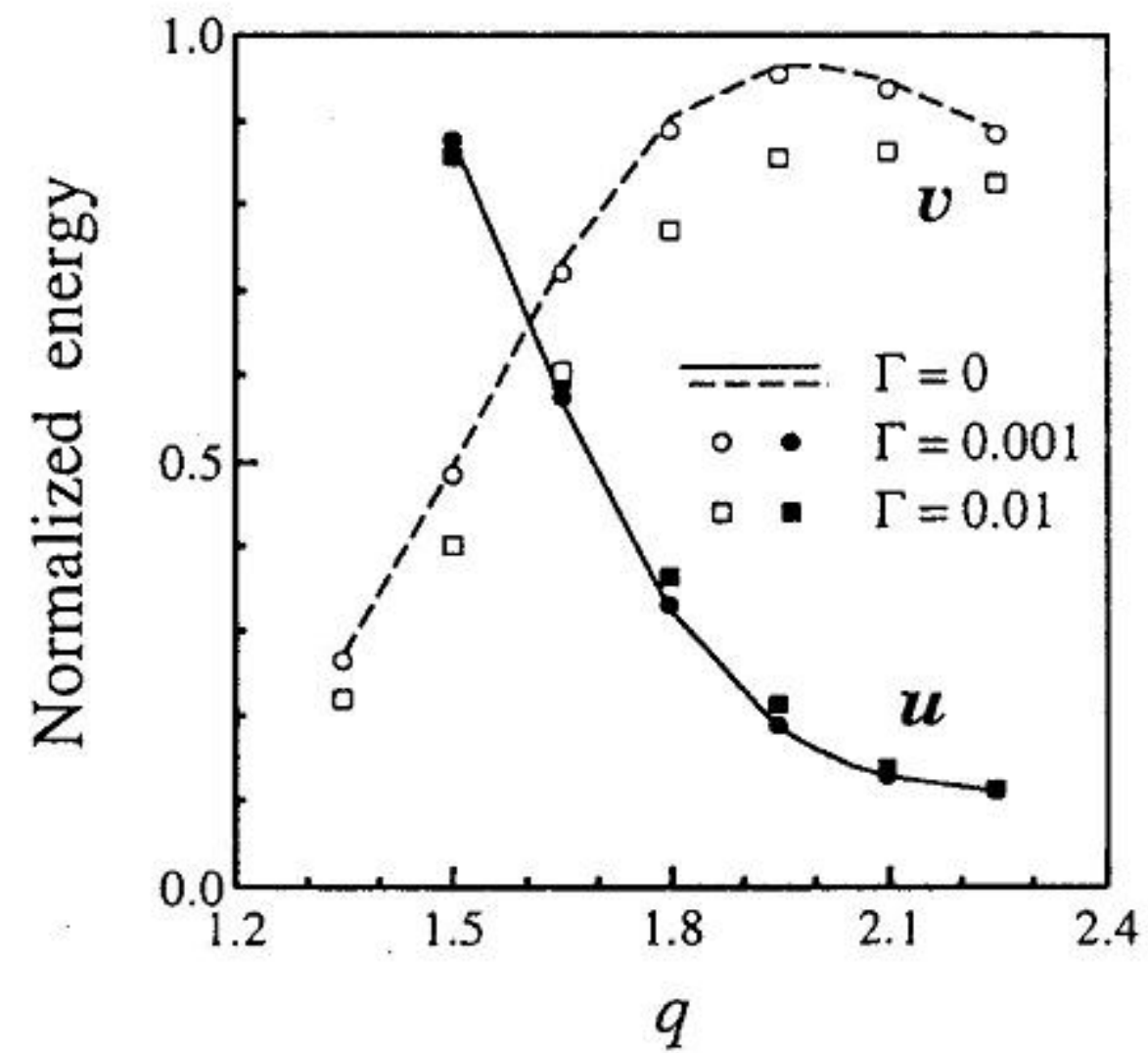

(b)

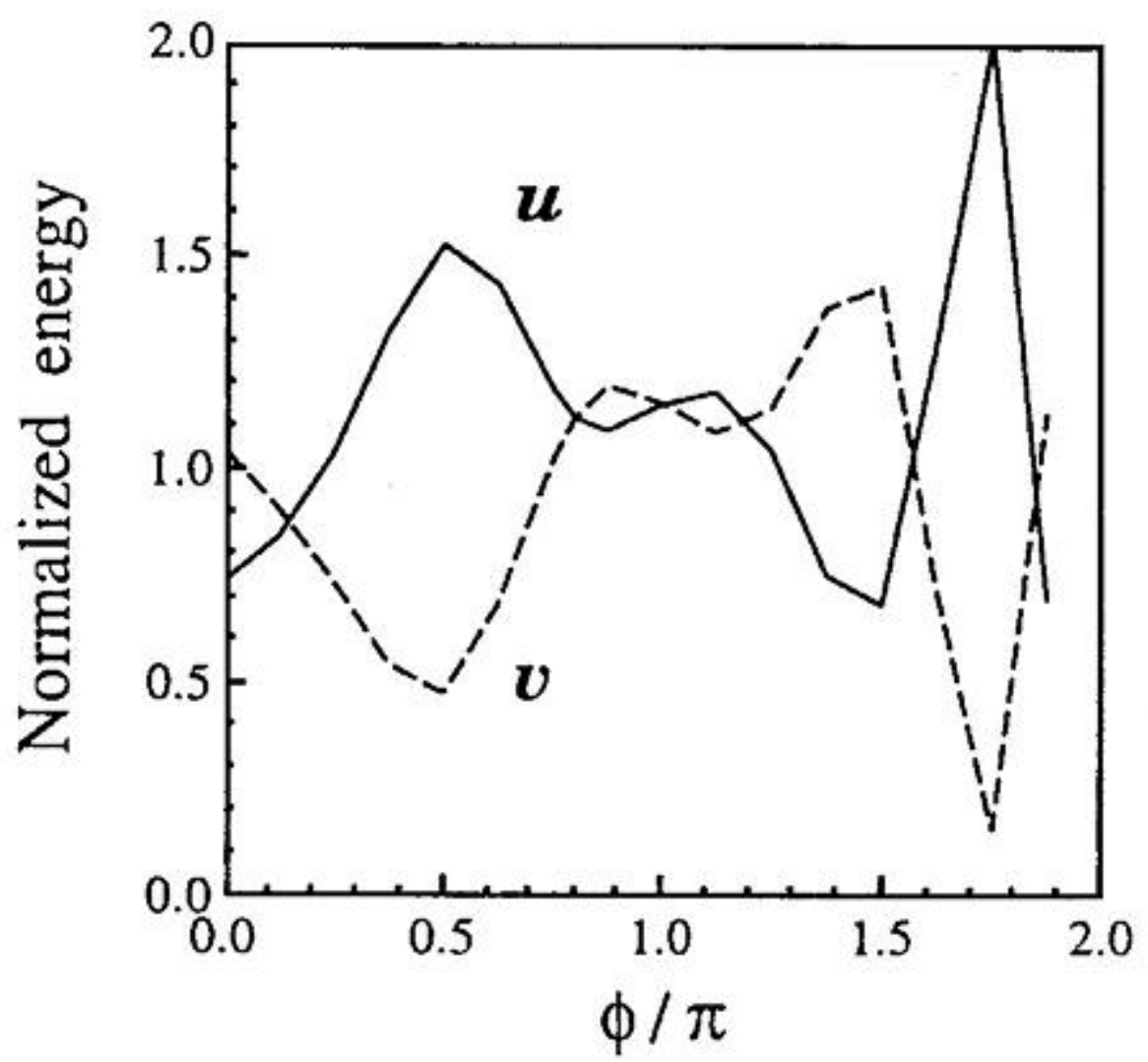

(c)

7ig. 5. Switching characteristics in the perturbed case using EDFA: (a) $A_{u}=1, A_{v}=0$, and $\phi=0$, (b) $A_{u}=0, A_{v}=1$, and $\phi=0$, and $A_{u}=A_{v}=1$ and $q=1.95$.

When using a fiber with $\sigma=0.45 \mathrm{ps} /(\mathrm{km} \cdot \mathrm{nm})$ and $\sigma_{\lambda}=0.06$ $\mathrm{ps} /\left(\mathrm{km} \cdot \mathrm{nm}^{2}\right)[2],[3]$, the coefficients related to the higher order effects are $\nu_{1}=0.20[22]-[24], \nu_{2}=0.01$, and $\nu_{3}=0.04$.

Fig. 2 shows the normalized energy at the coupling length, Where the results for lossy cases are also plotted by circles and quares and are discussed later (in Section IV-D). In Fig. 2(a) or (b), the fundamental soliton is inputted to $u$ - or $v$-channel, respectively, and we assume $\phi=0$. The contrast of output evels is degraded when the soliton is inputted to $v$-channel. The results shown in Fig. 2 are almost the same as those obtained by taking only TOD into account; $\nu_{1}=0.20$ and $\nu_{2}=\nu_{3}=0$, and therefore, the above contrast degradation of output levels is mainly due to TOD.

Fig. 3 shows the waveform changes when the optical soliton 18 inputted to $v$-channel, where $q=2.0$. It is found that small pulses are generated in the slow group velocity region In consequence of radiation caused by TOD, and that the contrast of output levels at the coupling length $\xi_{c} \simeq 1.5 \pi$ is degraded. Fig. 4 shows the spectrum changes, where $\omega_{0}$ is the carrier frequency, and $T=T_{\mathrm{FWHM}} / 1.762724$. It can be seen that the spectrum corresponding to the radiation is generated at a frequency $\left(\omega-\omega_{0}\right) T \simeq 1 / 2 \nu_{1}$ [25], and that the spectrum corresponding to the soliton component shifts to lower frequency region in consequence of SSFS.

In the present case, the coupling length given by $L_{c}=$ $Z_{s} / \kappa$ becomes $166 \mathrm{~m}$. When picosecond pulses are used, the coupling length increases, and the coefficient related to the higher order effects decrease. For example, when a 1 ps pulse is used, we obtain $L_{c}=2661 \mathrm{~m}, \nu_{1}=0.05, \nu_{2}$ $=3.0 \times 10^{-3}$ and $\nu_{3}=0.01$. If higher dispersion fibers are used, the coupling length and the TOD coefficient are reduced compared with the case of using lower-dispersion fibers. For example, when a fiber with $\sigma=3.0 \mathrm{ps} /(\mathrm{km} \cdot \mathrm{nm})$ and $\sigma_{\lambda}=0.05 \mathrm{ps} /\left(\mathrm{km} \cdot \mathrm{nm}^{2}\right)[20]$ is used, we obtain $L_{c}=25$ 


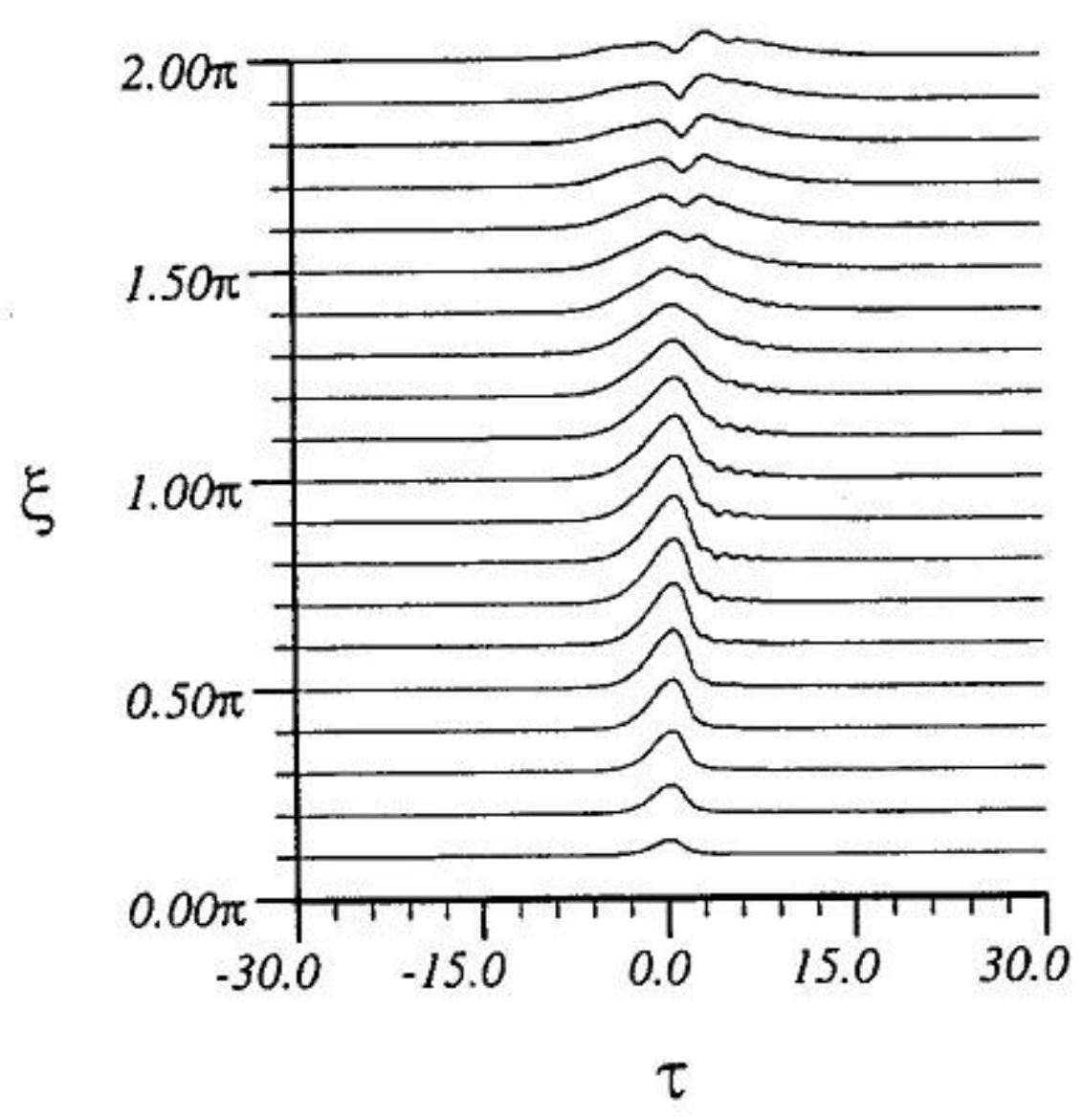

(a)

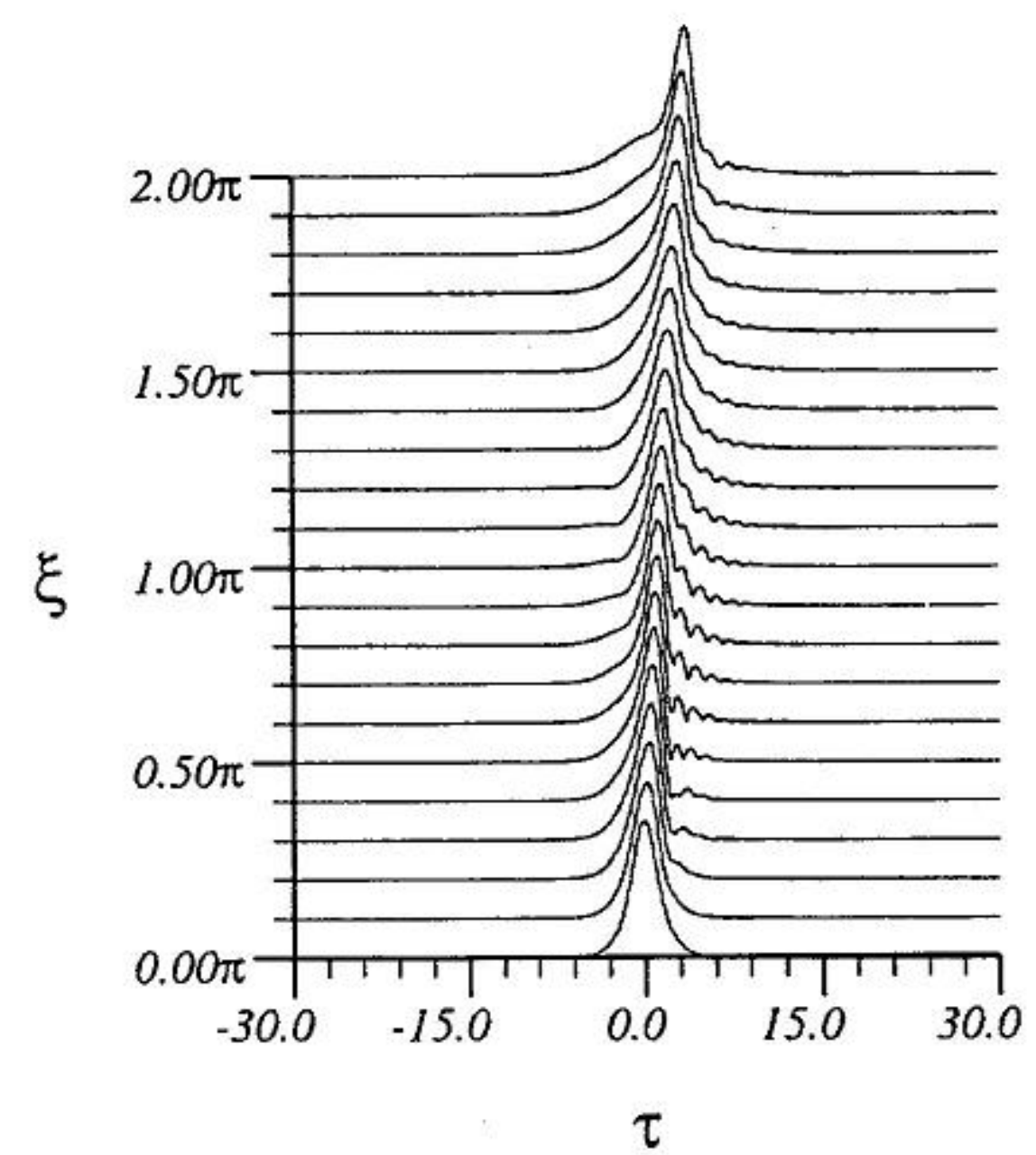

(b)

Fig. 6. Waveform changes in the perturbed case using EDFA $\left(A_{u}=0, A_{v}=1\right.$, and $\left.\phi=0\right)$ : (a) $u$-channel and (b) $v$-channel.

$\mathrm{m}$ and $\nu_{1}=0.025$ for a $0.25 \mathrm{ps}$ pulse. The values of $\nu_{2}$ and $\nu_{3}$ are unchanged for the same pulse-width.

\section{Soliton Trapping by EDFA}

In order to remove the spectrum corresponding to radiation caused by TOD, we use a distributed EDFA and try to trap only the soliton components in the limited bandwidth of the amplifier [22]-[24], [26]-[28].

Now, we assume the gain and the bandwidth of EDFA as $G=0.07$ and $\mu=1.5$, respectively. Fig. 5 shows the normalized energy at the coupling length. In contrast to Fig. 2 without EDFA, the contrast of output levels is improved with $q=1.95$ when a soliton is inputted to $v$-channel as shown in Fig. 5(b). It is found from Fig. 5(c) that the output

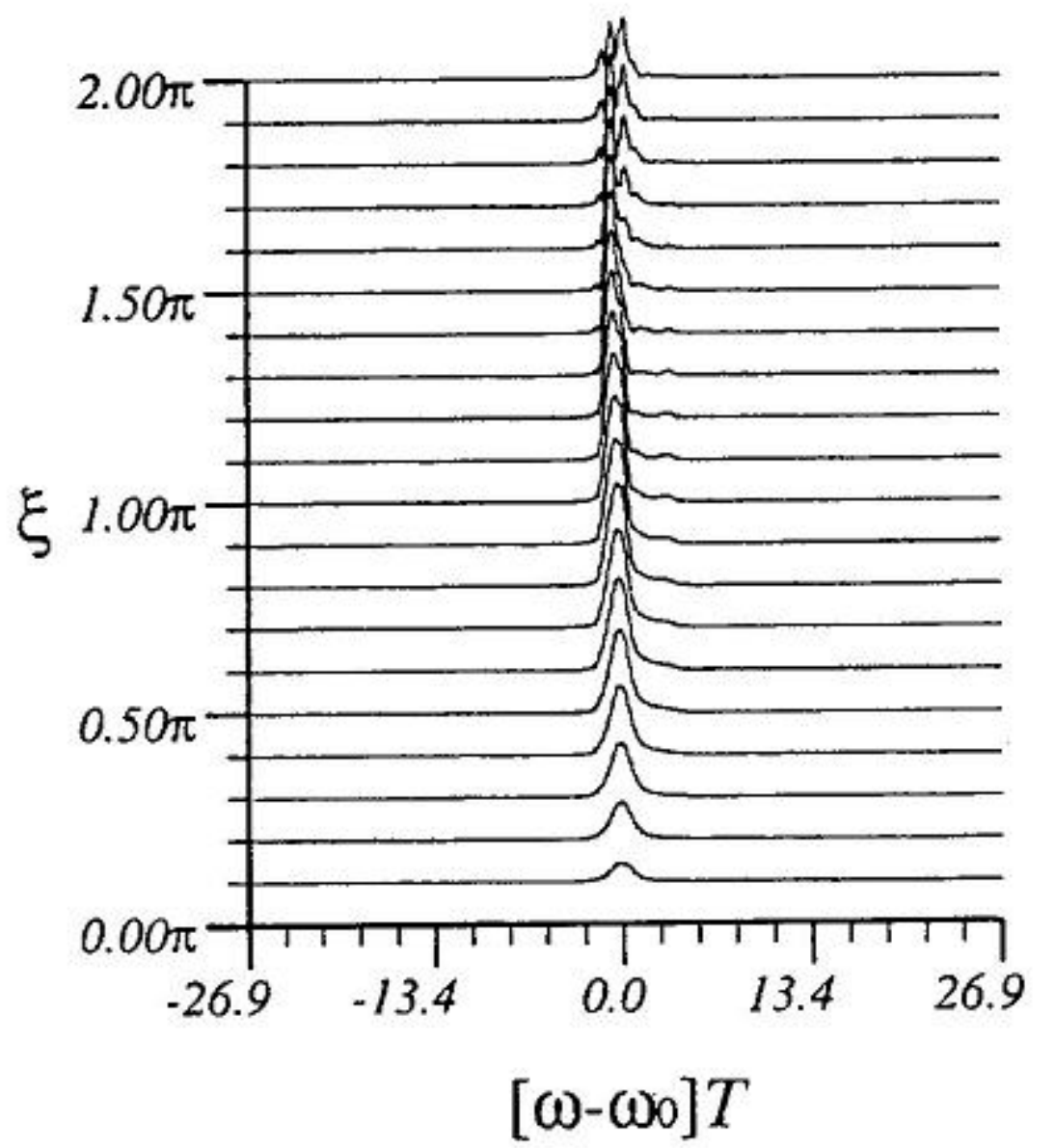

(a)

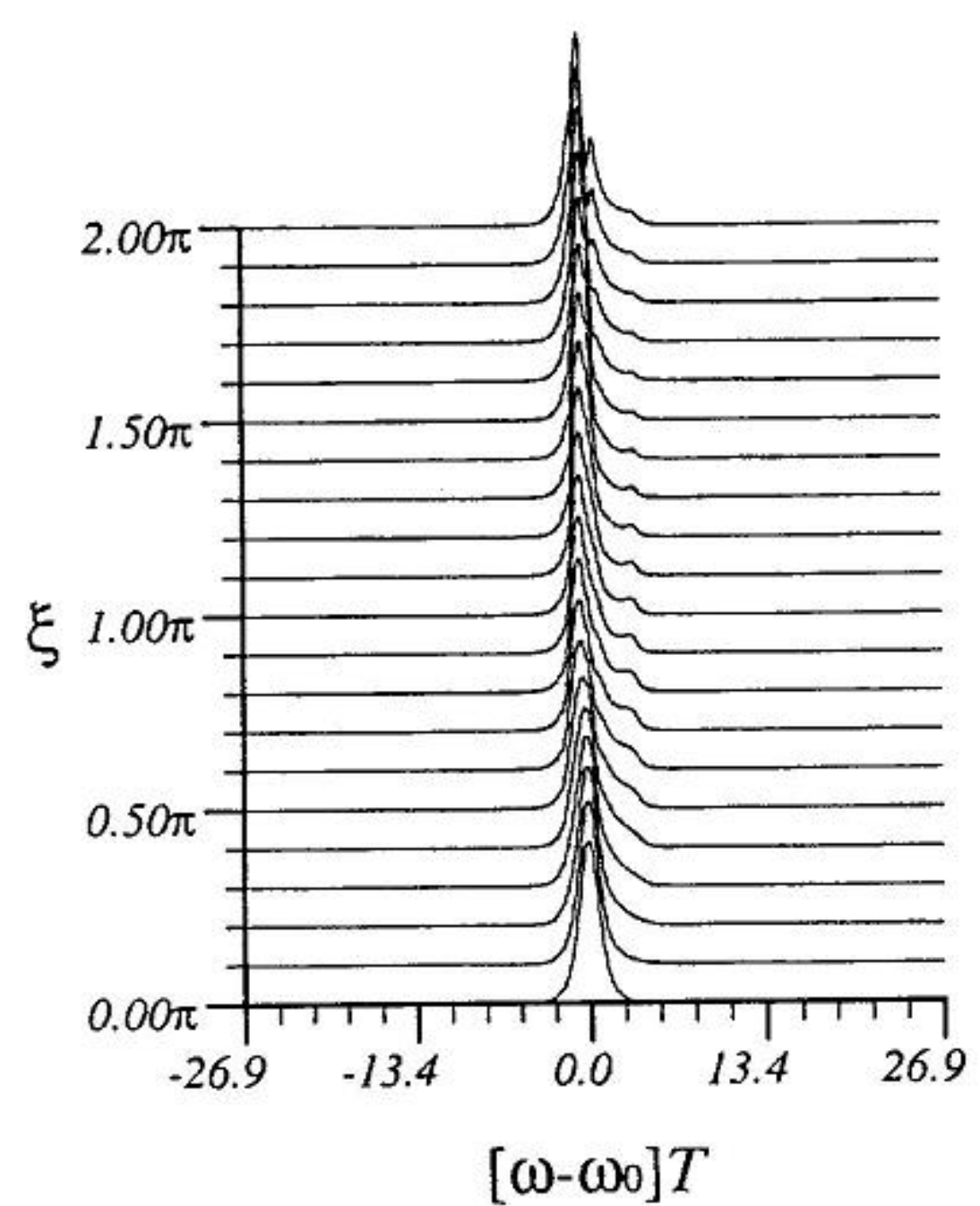

(b)

Fig. 7. Spectrum changes in the perturbed case using ( $A_{u}=0, A_{v}=1$, and $\phi=0$ ): (a) $u$-channel and (b) $v$-channel.

levels in $u$ - and $v$-channels are almost the same when $\phi=$ $0.800 \pi, 1.000 \pi, 1.200 \pi$, and $1.580 \pi$.

Fig. 6 shows the waveform changes when the optical soliton is inputted to $v$-channel, where $q=1.95$. It can be seen that the contrast of output levels at the coupling length $\xi_{c} \simeq 1.5 \pi$ is improved, and that the waveforms are less distorted. Fig.? shows the spectrum changes. The spectrum corresponding to the radiation caused by TOD is supressed.

When $G$ increases, the energy in $u$-channel increases more than in $v$-channel. And when $G$ decreases, the energy in $v$-channel does not increase compared with the energy in $u$ channel, and thus, the contrast of output levels deteriorates. When $\mu$ increases, the bandwidth of the amplifier is narrowed, 


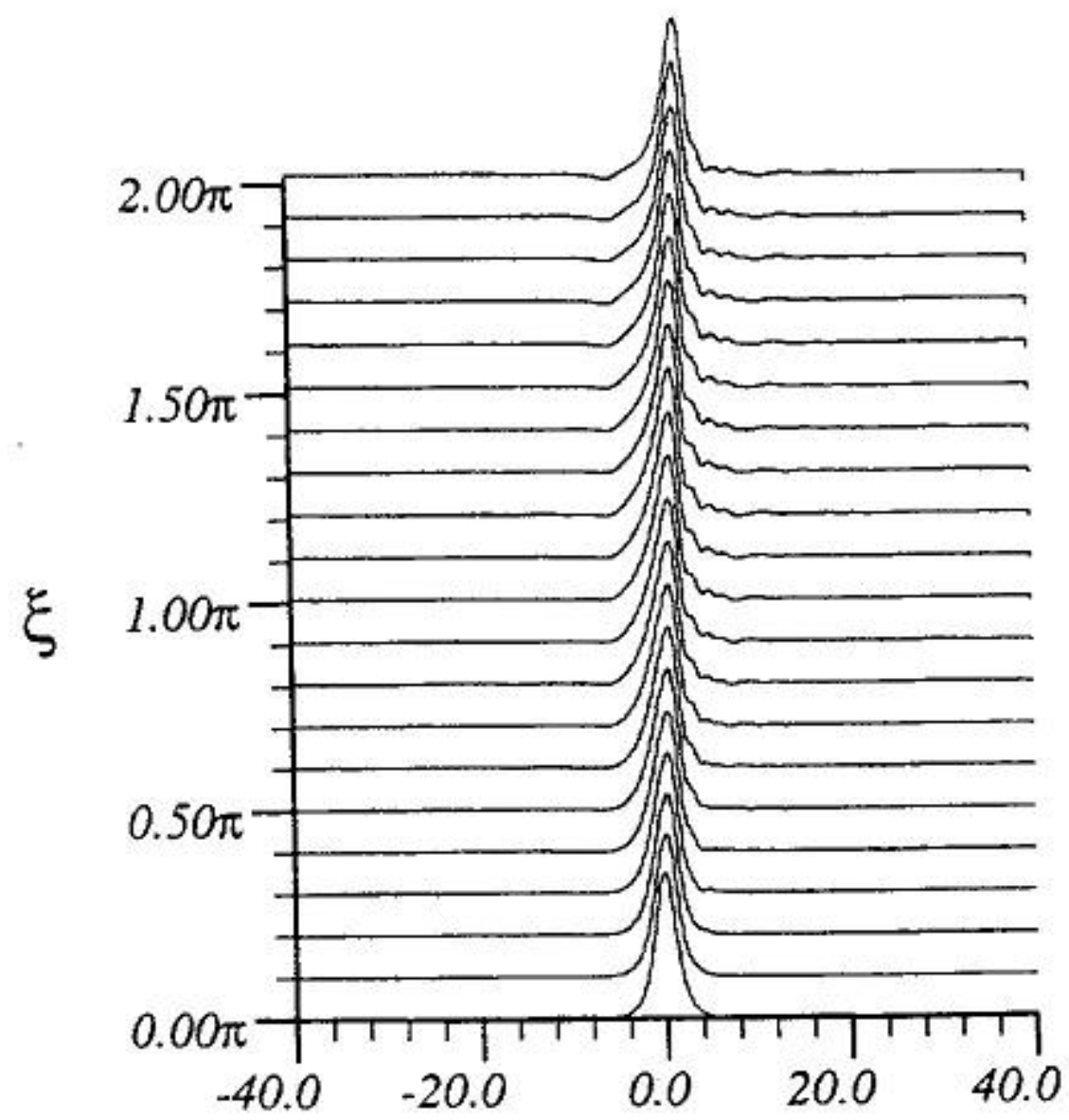

$\tau$

(a)

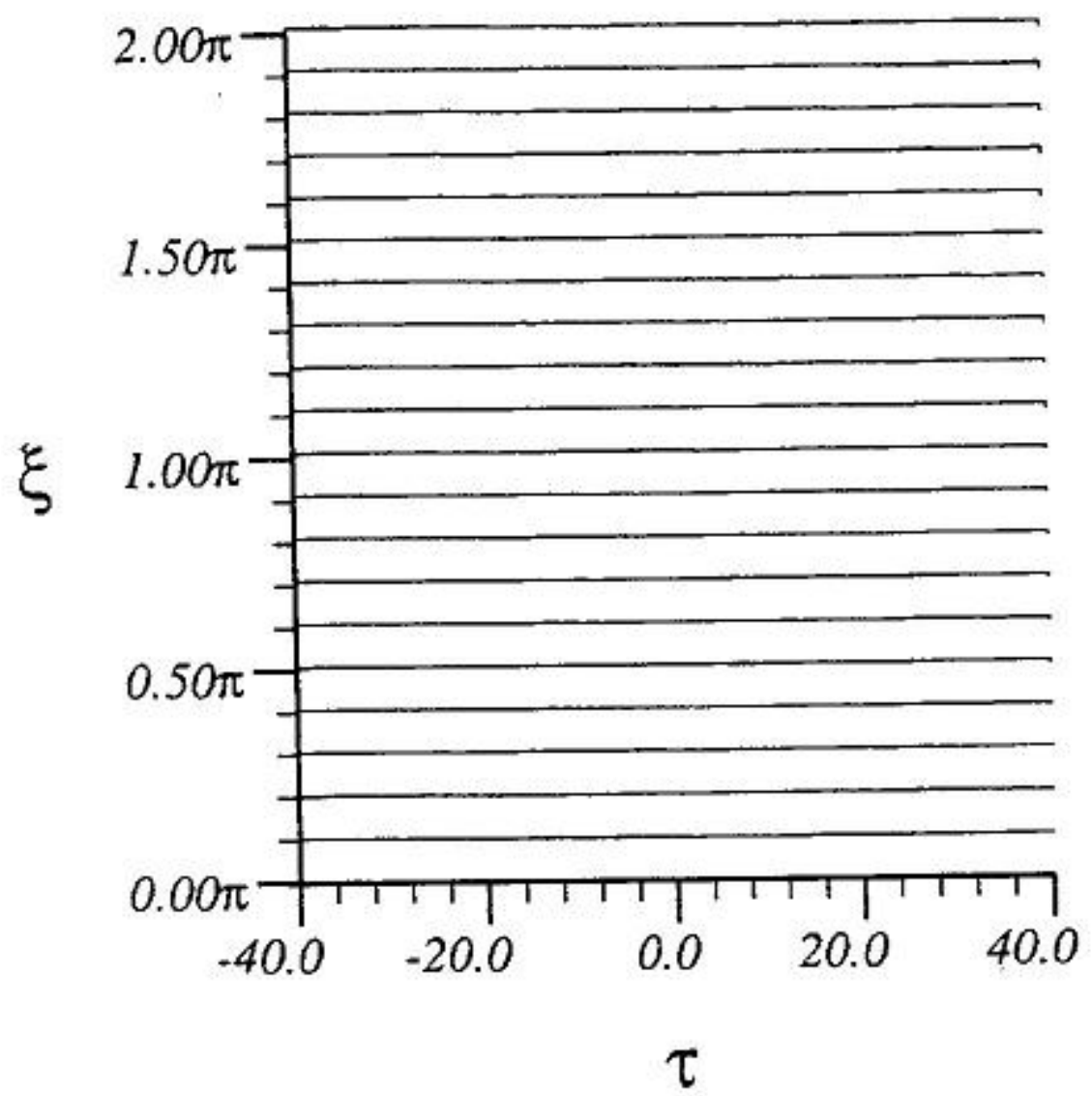

(b)

Fig. 8. Waveform changes in the perturbed case using EDFA with walk-off effect $\left(A_{u}=1, A_{v}=0\right.$, and $\left.\phi=0\right)$ : (a) $u$-channel,(b) $v$-channel.

and even the spectrum corresponding to the soliton component is partially cut out. When $\mu$ decreases, on the other hand, the bandwidth of the amplifier is widened, and the spectra corresponding to the radiation are also amplified. Therefore, the values of $G$ and $\mu$ should be carefully chosen.

As shown in Table I, the operation of logic devices can be improved by using bandwidth limited amplification regardless of TOD.

\section{Fiber Loss}

Here, we investigate the effect of fiber losses and assume $a=0.25 \mathrm{~dB} / \mathrm{km}$. The attenuation coefficient, $\Gamma$, included in coupled NLSE's becomes 0.001 for the parameters used in the

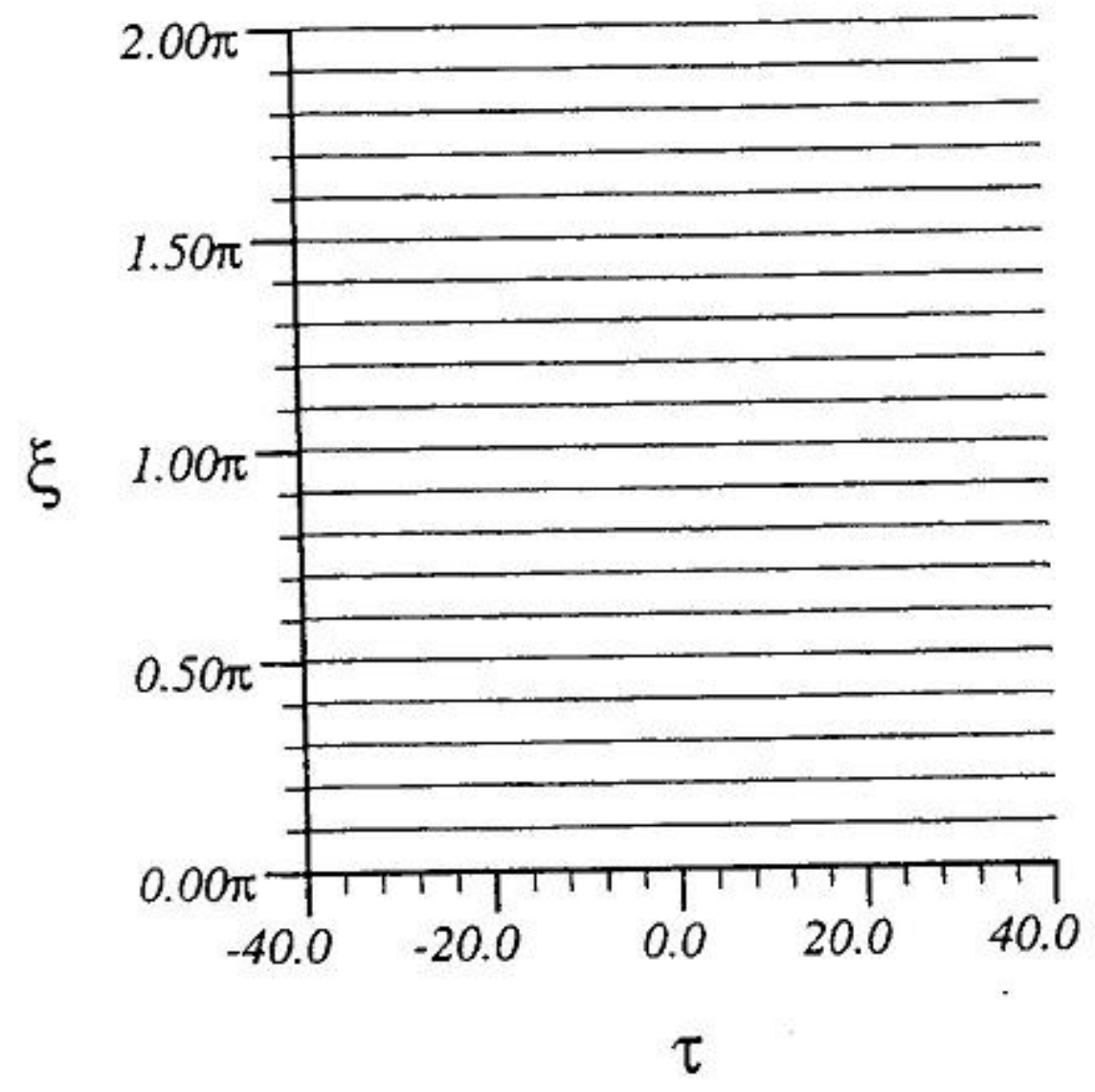

(a)

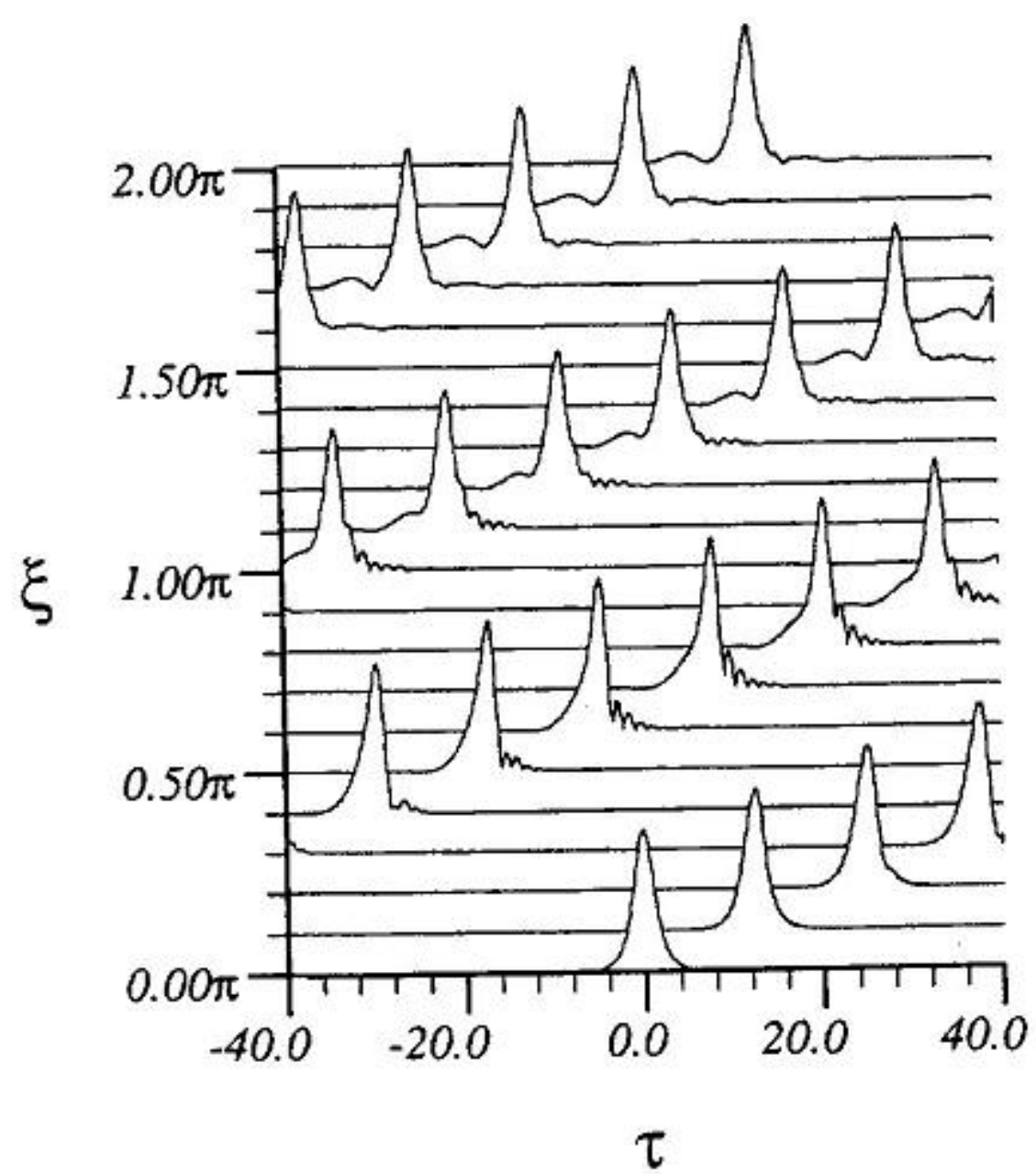

(b)

Fig. 9. Waveform changes in the perturbed case using EDFA with walk-off effect $\left(A_{u}=0, A_{v}=1\right.$, and $\left.\phi=0\right):$ (a) $u$-channel and (b) $v$-channel.

present calculation. In Figs. 2 and 5, the results for $\Gamma=0.001$ are plotted by closed and opened circles, and those for an artificially larger attenuation value $\Gamma=0.01$ are also plotted by closed and opened squares. When $\Gamma=0.001$, the switching characteristics are almost the same as the lossless case, and EDFA may be effectively utilized. When $\Gamma=0.01$, on the other hand, the switching characteristics are degraded, and the operation of logic devices is not always improved by EDFA.

\section{E. Walk-Off Effect}

When $q>1$, the core radius of $v$-channel is larger than that of $u$-channel. Assuming the relative index defference of $0.55 \%$, for example, the core radii of $u$ - and $v$-channels are, 
TABLE II

WALK-OFF EFFECTS ON LOGIC FUnCTIONS

\begin{tabular}{ccccc}
\hline \multirow{2}{*}{ Walk-off effect $\delta$} & \multicolumn{2}{c}{ Input } & \multicolumn{2}{c}{ Output } \\
\cline { 2 - 5 } & $E_{u}$ & $E_{v}$ & $E_{u}$ & $E_{v}$ \\
\hline 0 & 0 & 0 & 0 & 0 \\
& 1 & 0 & $0(0.22)$ & $1(1.19)$ \\
& 0 & 1 & $0(0.19)$ & $1(0.96)$ \\
& 1 & 1 & $1(1.12)$ & $1(1.12)$ \\
\hline-0.1 & 0 & 0 & 0 & 0 \\
& 1 & 0 & $0(0.21)$ & $1(1.20)$ \\
& 0 & 1 & $0(0.24)$ & $1(0.91)$ \\
& 1 & 1 & $1(0.95)$ & $1(1.01)$ \\
\hline-1.0 & 0 & 0 & 0 & 0 \\
& 1 & 0 & $0(0.22)$ & $1(1.26)$ \\
& 0 & 1 & $0(0.74)$ & $1(0.42)$ \\
& 1 & 1 & $1(0.79)$ & $1(1.21)$ \\
\hline & 0 & 0 & 0 & 0 \\
-10.0 & 1 & 0 & $0(1.25)$ & $1(0.22)$ \\
& 0 & 1 & $0(0.17)$ & $1(0.78)$ \\
& 1 & 1 & $1(1.43)$ & $1(1.04)$ \\
\hline
\end{tabular}

respectively, 1.46 and $2.42 \mu \mathrm{m}$ for $q=1.95$ used in Fig. 6 . In this case, from (11) the walk-off effect $\delta=-4368.5$ $\left(T_{d}=5.0 \times 10^{-3}\right)$, and GVD's of $u$ - and $v$-channels are almost the same. Fig. 8 shows the waveform changes when the optical soliton is inputted to $u$-channel. Higher order effects and EDFA are also included. Due to the walk-off effect, the optical soliton inputted to $u$-channel is not coupled with $v$ channel. Fig. 9 shows the waveform changes when the optical soliton is inputted to $v$-channel. Although the soliton inputted to $v$-channel remains in $v$-channel as in the case without the walk-off effect, the pulse shifts to the slow group velocity region. It is found from Figs. 8 and 9 that the walk-off effect makes it difficult to improve the operation of logic devices by EDFA.

When $q>1$, the walk-off effect $\delta$ becomes negative. Table II shows the switching characteristics for different values of the walk-off effect. Higher order effects and EDFA are included. The operation of logic devices is not affected for $\delta=-0.1$, but degraded for $\delta=-1.0$. So, the walk-off effect can be suppressed by suitably designing fiber parameters, and logic devices could be constructed by asymmetric nonlinear optical fiber couplers.

\section{CONCLUSION}

We investigated the effects of TOD, shock effect, and SSFS on the operation of logic devices constructed by asymmetric nonlinear optical fiber couplers. It was found that in consequence of the presence of TOD, the logic functions are degraded. In order to remove the influence of TOD, a distributed EDFA with a finite bandwidth was introduced, and the logic functions were improved by trapping only soliton components in the finite gain-bandwidth. The effects of fiber loss and walk-off were also investigated.

\section{REFERENCES}

[1] A. Hasegawa and F. Tappert, "Transmission of stationary nonlinear optical pulse in dispersive dielectric fibers, I. Anomalous dispersion;" Appl. Phys. Lett., vol. 23, pp. 142-144, Aug. 1973.

[2] L. F. Mollenauer, E. Lichtman, M. J. Neubelt, and G. T. Harvey; "Demonstration, using sliding-frequency guiding filters, of error-free" soliton transmission over more than $20 \mathrm{Mm}$ at $10 \mathrm{Gbit} / \mathrm{s}$, single channel, and over more than $13 \mathrm{Mm}$ at $20 \mathrm{Gbit} / \mathrm{s}$ in a two-channel WDM, Electron, Lett, vol. 29, pp. 910-911, May 1993.

[3] W. Forysiak and N. J. Doran, "Conjugate solitons in amplified optical fiber transmission systems," Electron. Lett., vol. 30, pp. 154-155, Jan 1994.

[4] M. Nakazawa, Y. Kimura, and K. Suzuki, "Soliton amplification and transmission with $\mathrm{Er}^{3+}$-doped fiber repeater pumped by GaInAsP lase diode," Electoron. Lett., vol. 25, pp. 199-200, Feb. 1989.

[5] G. P. Agrawal, Nonlinear Fiber Optics. New York: Academic, 1989,

[6] S. Trillo, S. Wabnitz, E. M. Wright, and G. I. Stegeman, "Soliton switching in fiber nonlinear directional couplers," Opt. Lett., vol. 13 pp. 672-674, Aug. 1988.

[7] E. Caglioti, S. Trillo, S. Wabnitz, B. Crosignali, and P. Di Porto, "Finite dimentional description of nonlinear pulse propagation in optical-fiber couplers with applications to soliton switching," J. Opt. Soc. Amer. B, vol. 7, pp. 374-385, Mar. 1990.

[8] C. Paré and M. Florjańczyk, "Approximate model of soliton dynamics in all-optical couplers," Phys. Rev. A, vol. 41, pp. 6287-6295, June 1990.

[9] J. Wilson, G. I. Stegeman, and E. M. Wright, "Soliton switching in an erbium-doped nonlinear fiber coupler," Opt. Lett., vol. 16, pp. 1653-1655, Nov. 1991.

[10] P. L. Chu, B. A. Malomed, and G. D. Peng, "Soliton switching and propagation in nonlinear fiber couplers: Analytical results," J. Opt. Soc Amer, B, vol. 10, pp. 1379-1385, Aug. 1993.

[11] G. L. Diankov, I. M. Uzunov, and F. Lederer, "Effect of third order dispersion on pulse dynamics in nonlinear directional couplers, Electoron. Lett., vol. 30, pp. 155-156, Jan. 1994.

[12] N. N. Akhmediev and A. V. Buryak, "Soliton states and bifurcation phenomena in three core nonlinear fiber couplers," J. Opt. Soc. Amer, $B$, vol. 11 , pp. 804-809, May 1994.

[13] H. Sotobayashi and Y. Fujii, "Optical soliton switching and coupled soliton propagation in three-core fiber nonlinear directional coupler," Trans. IEICE, vol. J78-C-I, pp. 157-165, Mar. 1995 (in Japanese).

[14] I. M. Uzunov, R. Muschall, M. Gölles, B. A. Malomed, and F. Lederer, "Pulse switching in nonlinear fiber directional couplers," Phys. Rev. E, vol. 51, pp. 2527-2537, Mar. 1995.

[15] A. V. Buryak and N. N. Akhmediev, "Stationary pulse propagation in $N$-core nonlinear fiber arrays," IEEE J. Quantum Electron., vol. 31, pp. 682-688, Apr. 1995.

[16] C. C. Yang, "All-optical ultrafast logic gates that use asymmetric nonlinear directional couplers," Opt. Lett., vol. 16, pp. 1641-1643, Nov, 1991.

[17] C. G. Krautschik, G. I. Stegeman, and R. H. Stolen, "Phase controlled" all-optical switching in rocking filter fibers," Appl. Phys. Lett., vol. 61, pp. 1751-1753, Oct. 1992.

[18] C. G. Krautschik, G. I. Stegeman, and R. H. Stolen, "Asymmetric" response of nonlinear coupled-mode devices: All-optical logic gates with a rocking-filter fiber," Opt. Lett., vol. 18, pp. 1050-1052, July 1993.

[19] P. Ferro, S. Trillo, and S. Wabnitz, "Demonstration of nonlinear nonreciprocity and logic operations with a twisted birefringent optical fiber," Opt. Lett., vol. 19, pp. 263-265, Feb, 1994.

[20] M. Nakazawa, H. Kubota, K. Kurokawa, and E. Yamada, "Femtosecond optical soliton transmission over long distance using adiabatic trapping and soliton standardization," J. Opt. Soc. Amer. B, vol. 8, pp. 1811-1817, Sept. 1991.

[21] M. Nakazawa and H. Kubota, "Physical interpretation of reduction of soliton interaction forces by bandwidth limited amplification," Electoron. Lett., vol. 28, pp. 958-960, May 1992.

[22] Y. Kodama, M. Romagnoli, S. Wabntiz, and M. Midrio, "Role of thirdorder dispersion on soliton instabilities and interactions in optical fibers," Opt. Lett., vol. 19, pp. 165-167, Feb. 1994.

[23] I. M. Uzunov, M. Gölles, and F. Lederer, "Soliton interaction in the presence of bandwidth limited amplification near the zero dispersion wavelength," Electron. Lett., vol. 30, pp. 882-884, May 1994.

[24] I. M. Uzunov, M. Gölles, and F. Lederer, "Soliton interaction near the zero-dispersion wavelength," Phys. Rev. E, vol. 52, pp. 1059-1071, July 1995.

[25] P. K. A. Wai, C. R. Menyuk, Y. C. Lee, and H. H. Chen, "Nonlinear pulse propagation in the neighborhood of the zero-dispersion wavelength. of monomode optical fibers," Opt. Lett., vol. 11, pp. 464-466, July 1986. 
26] F. S. Locatti, M. Romagnoli, A. Tajani, and S. Wabntiz, "Adiabatic femtosecond soliton active nonlinear directional coupler," Opt. Lett., vol. 17, pp. 1213-1315, Sept. 1992.

127] A. B. Aceves, C. De Angelis, G. Nalesso, and M. Santagiustina, "Higher-order effects in bandwidth-limited soliton propagation in optical fiber," Opt. Lett., vol. 19, pp. 2104-2106, Dec. 1994.

[28] D. Shenoy and A. Puri, "Compensation for the soliton self-frequency shift and the third-order dispersion using bandwidth-limited optical gain," Opt. Comm, vol. 113, pp. 401-406, Jan. 1995.

Masanori Koshiba (SM'84) was born in Sapporo, Japan, on November 23, 1948. He received the B.S., M.S., and Ph.D. degrees in electronic engineering from Hokkaido University, Sapporo, Japan, in 1971, 1973, and 1976 , respectively.

In 1976, he joined the Department of Electronic Engineering, Kitami Institute of Technology, Kitami, Japan. From 1979 to 1987, he was an Associate Professor of Electronic Engineering at Hokkaido University, and in 1987, he became a Professor. He has been engaged in research on lightwave technology, microwave field theory, surface acoustic waves, magnetostatic waves, electron waves, CAD for photonic devices, and applications of finite ilement and boundary element methods to field problems. He is an author tor coauthor of more than 200 research papers in refereed journals. He is an thuthor of the books, Optical Waveguide Analysis (New York: McGraw-Hill) and Optical Waveguide Theory by the Finite Element Method (New York: KTK Scientific/Kluwer Academic) and is a coauthor of the book, Analysis Methods for Electromagnetic Wave Problems (Norwood, MA: Artech House).

Dr. Koshiba is a member of the Institute of Electronics, Information and Communication Engineers (IEICE) of Japan, the Institute of Electrical Engineers of Japan, the Institute of Television Engineers of Japan, the Japan Society for Simulation Technology, the Japan Society for Computational Methods in Engineering, and the Japan Society of Applied Electromagnetics. In 1987, he was awarded the 1986 Excellent Paper Award from the IEICE.
Takashi Kojima was born in Sapporo, Japan, on March 28, 1972. He received the B.S. degree in electronic engineering from Hokkaido University, Sapporo, Japan, in 1994. He is presently working toward the M.S. degree in electronic engineering at Hokkaido University.

Mr. Kojima is a member of the Institute of Electronics, Information and Communication Engineers (IEICE) of Japan.

Yasuhide Tsuji was born in Takikawa, Hokkaido, Japan, on December 31, 1967. He received the B.S. and M.S. degrees in electronic engineering from Hokkaido University, Sapporo, Japan, in 1991 and 1993, respectively. He is presently working toward the Ph.D. degree in electronic engineering at Hokkaido University.

Mr. Tsuji is a member of the Institute of Electronics, Information and Communication Engineers (IEICE) of Japan.

Masashi Eguchi (M'93) was born in Sapporo, Japan, on August 9, 1962. He received the B.S. degree in electronic engineering from Kitami Institute of Technology, Kitami, Japan, in 1985 and the M.S. and Ph.D. degrees in electronic engineering from Hokkaido University, Sapporo, Japan, in 1987, 1991 , respectively.

He joined the Sony Co., Ltd., in 1987. From 1991 to 1995 , he was with the Department of Industrial Design at Sapporo School of the Arts, Sapporo, Japan. Since 1995, he has been an Instructor of Electrical and Electronic Engineering at Muroran Institute of Technology, Muroran, Japan. He has been engaged in research on optical fibers, dielectric waveguides, optical solitons and applications of finite element method.

Dr. Eguchi is a member of the Institute of Electronics, Information and Communication Engineers (IEICE) of Japan. 